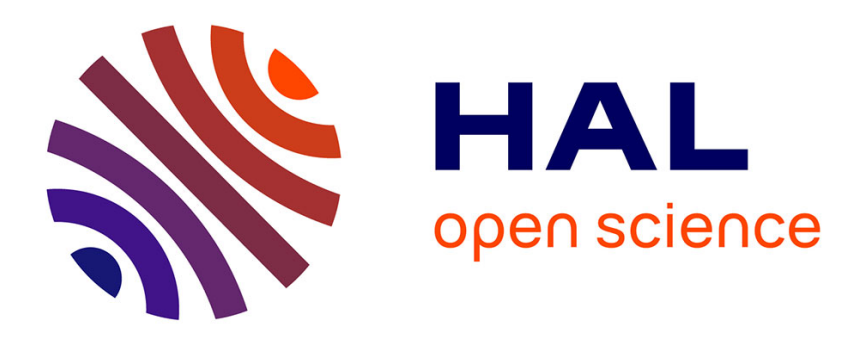

\title{
Graph Theory in Coq: Minors, Treewidth, and Isomorphisms
}

Christian Doczkal, Damien Pous

\section{To cite this version:}

Christian Doczkal, Damien Pous. Graph Theory in Coq: Minors, Treewidth, and Isomorphisms. Journal of Automated Reasoning, 2020, 10.1007/s10817-020-09543-2 . hal-02316859v2

\section{HAL Id: hal-02316859 \\ https://hal.science/hal-02316859v2}

Submitted on 19 Jan 2020

HAL is a multi-disciplinary open access archive for the deposit and dissemination of scientific research documents, whether they are published or not. The documents may come from teaching and research institutions in France or abroad, or from public or private research centers.
L'archive ouverte pluridisciplinaire HAL, est destinée au dépôt et à la diffusion de documents scientifiques de niveau recherche, publiés ou non, émanant des établissements d'enseignement et de recherche français ou étrangers, des laboratoires publics ou privés. 


\title{
Graph Theory in Coq: Minors, Treewidth, and Isomorphisms ${ }^{\star}$
}

\author{
Christian Doczkal · Damien Pous
}

December 18, 2019

\begin{abstract}
We present a library for graph theory in Coq/Ssreflect. This library covers various notions on simple graphs, directed graphs, and multigraphs. We use it to formalize several results from the literature: Menger's theorem, the excludedminor characterization of treewidth-two graphs, and a correspondence between multigraphs of treewidth at most two and terms of certain algebras.
\end{abstract}

Keywords graph theory, minor, treewidth, isomorphisms, Coq, Ssreflect

\section{Introduction}

Despite the importance of graph theory in mathematics and computer science, there are only a few formalizations of graph theory results in interactive theorem provers, and even fewer general purpose libraries.

In the 1990s, Chou formalized some basic undirected graph theory (paths, connectedness, trees) in HOL [2] and used it for the verification of distributed algorithms [3]. Nakamura and Rudnicki formalized Euler's theorem in Mizar [25]. In the 2000s, several authors formalized results about planar graphs: in addition to Gonthier's celebrated formal proof of the Four-Color Theorem [17], planar graphs were formalized in Isabelle/HOL for the Flyspeck project [26] and Durfourd and Bertot employed a notion of graphs based on hypermaps embedded in a plane to study Delaunay triangulations [14]. More recently, Noschinski developed a library

* This paper extends and revises the results presented in [10]; the underlying Coq library is available from https://perso.ens-lyon.fr/damien.pous/covece/graphs/.

This work has been funded by the European Research Council (ERC) under the European Union's Horizon 2020 programme (CoVeCe, grant agreement No 678157), and was supported by the LABEX MILYON (ANR-10-LABX-0070) of Université de Lyon and UCA ${ }^{\text {JEDI }}$, within the programs "Investissements d'Avenir" ANR-11-IDEX-0007 and ANR-15-IDEX-01, respectively.

C. Doczkal

Université Côte d'Azur, Inria Sopia Antipolis Méditerranée, France

D. Pous

Univ Lyon, CNRS, ENS de Lyon, UCB Lyon 1, LIP, France 
for both simple and multigraphs in Isabelle/HOL [27]. Singh and Natarajan have also formalized a proof of the weak perfect graph theorem in Coq, using Lovász replication lemma [32].

We propose in the present paper a general purpose graph library for Coq. The library deals with simple graphs, directed graphs, and multigraphs; it currently includes basic notions like paths, trees, subgraphs, separators, and isomorphisms, as well as a few more advanced ones: minors, and treewidth, whose theory was never formalized to the best of our knowledge. We use the library to formalize three distinct results, which we discuss below.

Menger's theorem. Menger's Theorem [24] states that if one needs to remove at least $n$ vertices to disconnect two sets of vertices $A$ and $B$ of some graph, then there exist $n$ pairwise disjoint paths from $A$ to $B$. Diestel [8, p. 50] calls Menger's Theorem [24] one of the cornerstones of graph theory and remarks that Hall's Marriage Theorem [20], a straightforward consequence of Menger's Theorem, is one of the most applied graph-theoretic results [8, p. 42].

Menger's Theorem provides a good test-case for our graph library: it admits a very short paper proof [18], but it nevertheless requires tools to work efficiently with several basic concepts like paths (including collections of paths), and deleting vertices and edges from graphs. We prove the theorem for directed graphs and use it to derive several corollaries on simple graphs as well as multigraphs: this ensures that its formulation is general enough and that our infrastructure makes it possible to transfer results between different kinds of graphs with minimal effort.

Excluded-minor characterization for treewidth one and two. The notion of treewidth [8] measures how close a graph is to a forest. Graph homomorphism (and thus $k$ coloring) becomes polynomial-time for classes of graphs of bounded treewidth [15, $1,19]$, and so does model-checking of monadic second-order (MSO) formulas; satisfiability of MSO formulas becomes decidable, even linear $[6,7]$.

Robertson and Seymour's graph minor theorem [30], a cornerstone of algorithmic graph theory, states that graphs are well-quasi-ordered by the minor relation. As a consequence, the classes of graphs of bounded treewidth, which are closed under taking minors, can each be characterized by finitely many excluded minors. While the graph minor theorem is nonconstructive and does not yield the excluded minors, low-width instances were known before: the graphs of treewidth at most one (the forests) are precisely those excluding the cycle with three vertices $\left(\mathrm{K}_{3}\right)$; those of treewidth at most two are those excluding the complete graph with four vertices $\left(\mathrm{K}_{4}\right)$ [13].
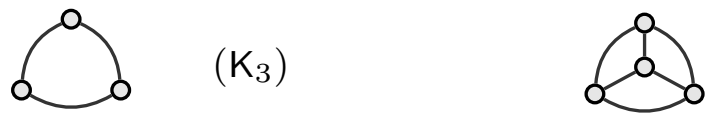

$\left(\mathrm{K}_{4}\right)$

Building on our library, we present constructive and formal proofs of both results in Coq/Ssreflect. Unlike in the conference version of this paper [10], we give here a direct proof of the excluded minor characterization of treewidth at most two relying on Menger's Theorem. 
Multigraphs of treewidth two seen as an algebra. Among the open problems related to treewidth, there is the question of finding finite axiomatizations of isomorphism for graphs of a given treewidth [7, page 118]. This question was recently answered positively for treewidth two $[23,11]$ :

Two-pointed $\mathrm{K}_{4}$-free multigraphs form the free $2 p$-algebra,

where $2 p$-algebras are algebraic structures characterized by twelve equational axioms. The proof is rather technical; it builds on a precise analysis of the structure of $\mathrm{K}_{4}$-free graphs. Further, invalid proofs of related claims have already been published in the literature (see [23]).

Our initial motivation for this work [10] was to formalize $(\dagger)$ : not only will this give us assurance about the validity of the proof in [23], it will also allow for the development of automation tactics for certain algebraic theories (e.g., 2p-algebra, allegories [16, 29]).

We present three important steps towards the formalization of this result:

1. graphs form a $2 \mathrm{p}$-algebra;

2. graphs of treewidth at most two form a subalgebra;

3. every graph excluding $\mathrm{K}_{4}$ as a minor can be represented by a term.

These three steps cover different situations which are frequently encountered when reasoning about graphs. The first point requires us to define several constructions on graphs (e.g., disjoint unions and quotients), and to establish a number of isomorphisms. The second point requires us to show that these constructions preserve treewidth. The third point requires us to analyze the structure of $\mathrm{K}_{4}$-free graphs in order to decompose them recursively; for this we again make use of Menger's theorem.

The final step for $(\dagger)$ consists in proving that terms denoting isomorphic graphs are equivalent modulo the axioms of $2 \mathrm{p}$-algebras. We formalized this step during the reviewing process of this article; the formalization is based on the proof in [11] rather than the one in [23] which we had set out to formalize in [10]. Proving $(\dagger)$ required us to introduce new techniques which we describe in [12]. We do not describe this work in the present article.

Outline. We discuss our representation choices for directed graphs, simple graphs, and paths in Section 3. Then we proceed to proving Menger's Theorem (Section 4). We define tree decompositions and minors in Sections 5 and 6 , so that we can prove the minor-exclusion theorem for treewidth two in Section 7. We end the presentation of the general purpose part of the library by defining labeled multigraphs in Section 8, together with the associated notion of isomorphism.

In the remaining sections we apply our library to $2 \mathrm{p}$-algebra: We define a $2 \mathrm{p}$ algebra of graphs and show that treewidth at most two graphs form a subalgebra (Section 9). We then prove a number of lemmas for analyzing the structure of $\mathrm{K}_{4}$ free graphs (Section 10), which make it possible to recursively extract terms from connected $\mathrm{K}_{4}$-free graphs (Section 11 ). We finally extend this extraction function to arbitrary $\mathrm{K}_{4}$-free graphs in Section 12 . 
Differences with [10]. The Coq library accompanying this paper [9] evolved significantly since [10] and continues to evolve, hence the different structure of the present paper: the main contribution is the graph library itself, rather than the application to 2 p-algebras.

The most noticeable improvement is that we prove Menger's Theorem and use it both to provide a direct proof of the minor-exclusion theorem for treewidth two, and to simplify the recursive analysis of $\mathrm{K}_{4}$-free graphs. (Lemma 10.8 follows easily with Menger's Theorem, while it was requiring a long series of ad-hoc lemmas in [10].) We also prove that graphs satisfy the laws of 2p-algebra (Lemmas 9.2 and 9.3). This required us develop techniques for dealing with the complicated isomorphisms arising with nested quotients and disjoint unions in a compositional manner (Lemma 8.7).

\section{Notation and Type-Theory Preliminaries}

If $X$ and $Y$ are types, we write $X+Y$ for the sum type (with elements inl $x$ and inr $y$ ), $X \times Y$ for the product type (with elements $(x, y)$ ), and $X_{\perp}$ for the option type (with elements Some $x$ and None). For indexed type families $T: X \rightarrow$ Type, we write $\Sigma(x: X) . T x$ for the sigma type (i.e., the type of dependent pairs) with elements $\langle x, y\rangle$ where $x: T$ and $y: T x$.

As usual, we write $g \circ f$ for the composition of two functions $f$ and $g$. For functions $f$ and $g$, we write $f \equiv g$ to mean that $f$ and $g$ agree on all arguments.

We use finite types as defined in the mathematical components library [34]. We briefly introduce them, together with the associated notations we are going to use in the mathematical development.

A finite type is a type $X$ together with a list enumerating its elements. Examples of finite types are the type $\mathbb{B}$ of booleans, and the type $I_{n}$ of natural numbers smaller than $n$. Finite types are closed under many type constructors. In particular, they are closed under sums, products, and sigma types. If $X$ is a finite type, we write $2^{X}$ for the finite type of sets over $X$ with decidable membership. For sets $A: 2^{X}$, we write $\bar{A}$ for complement of $A$ in $X$. We slightly abuse notation and also write $X$ for the full set over some type $X$. In particular, we use |-| to denote the cardinality of both sets and finite types (e.g., $\left|I_{n}\right|=n$ ). Finite sets come with an operation pick : $2^{X} \rightarrow X_{\perp}$ where pick $A=$ Some $x$ for some $x \in A$ if $A$ is nonempty and pick $A=$ None otherwise. If $\approx: X \rightarrow X \rightarrow \mathbb{B}$ is a boolean equivalence relation, the quotient [4] of $X$ with respect to $\approx$, written $X / \approx$, is a finite type as well. The type $X / \approx$ comes with functions $\pi: X \rightarrow X_{/} \approx$ and $\bar{\pi}: X / \approx \rightarrow X$ such that $\pi(\bar{\pi} x)=x$ for all $x: X / \approx$ and $\bar{\pi}(\pi x) \approx x$ for all $x: X$.

\section{Graphs and Paths}

We use finite types as the basic building block for defining (finite) graphs. ${ }^{1}$

Definition 3.1 A (finite) directed graph, or digraph for short, is a structure $\langle V, R\rangle$ where $V$ is a finite type of vertices and $R: V \rightarrow V \rightarrow \mathbb{B}$ is a decidable (i.e., boolean)

1 Most of the "Definition" and "Lemma" and "Theorem" headers in this paper are links to the corresponding entity in the Coq development. 
edge relation. A simple graph is a digraph whose edge relation is symmetric and irreflexive.

In Coq, we represent finite digraphs and simple graphs using dependently typed records:

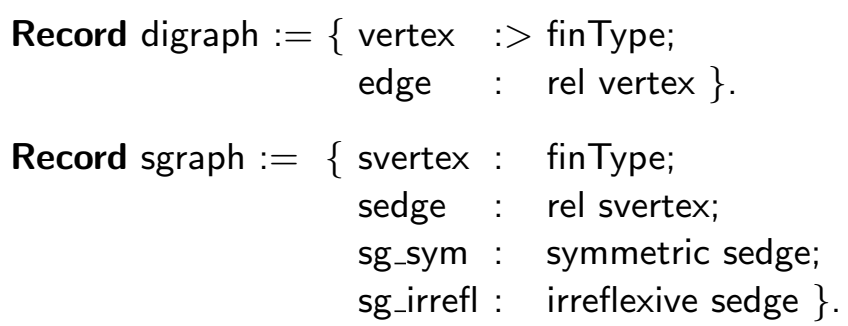

Note that for simple graphs two of the components are propositions. We introduce a coercion from simple graphs to digraphs forgetting symmetry and reflexivity of the edge relation. This allows us to define notations and notions like paths on digraphs, the class of graphs with the least structure, and have simple graphs inherit these notations and notions through the coercion. Declaring the vertex field of digraphs as a coercion, allows us to write $x: G$ to denote that $x$ is a vertex of $G$ and $|G|$ to denote the number of vertices of $G$ (irrespective of whether $G$ is a digraph or a simple graph). For vertices $x, y: G$ we write $x-y$ if there is an edge between $x$ and $y$. We write $G+x y$ for the graph $G$ with an additional $x y$-edge and $G-x y$ for $G$ with any potential $x y$-edge removed.

For a set $U: 2^{G}$ of vertices of $G$, the subgraph induced by $U$, written $\left.G\right|_{U}$, has as vertices the vertices in $U$ and as edge relation the edge relation of $G$ restricted to $U$. This is formalized by taking $\Sigma x: G . x \in U$ as the type of vertices ${ }^{2}$ and lifting the edge relation accordingly. While, technically, the vertices of $G$ and $\left.G\right|_{U}$ have different types, we will ignore this in the mathematical presentation. In Coq, we have a generic projection from $\left.G\right|_{U}$ to $G$. For the converse direction we, of course, need to construct dependent pairs of vertices $x: G$ and proofs of $x \in U$.

Remark 3.2 The constructions $\left.G\right|_{U}$ and $G+x y$ (for a given graph $G$ ) behave very differently in proofs. As mentioned above, the type of vertices of $\left.G\right|_{U}$ is different from the type of vertices of $G$. This is not the case for $G+x y$ (or any construction changing only the edge relation). As a consequence, $x: G$ iff $x: G+x y$ and likewise for sets or lists of vertices. This makes an explicit conversion of the vertices unnecessary.

Almost every argument in graph theory involves paths in one form or another. Consequently, finding the right representation of paths is of utmost importance when formalizing graph theory.

Definition 3.3 Let $G$ be a digraph. An $x y$-path is a nonempty sequence of vertices $p$ beginning with $x$ and ending with $y$ such that $z-z^{\prime}$ for all adjacent elements $z$ and $z^{\prime}$ of $p$ (if any). A path is irredundant if all vertices on the path are distinct.

2 To be fully precise, we use the type $\Sigma x: G . x \in U=$ true, exploiting that set membership is decidable. Since equality between booleans is proof irrelevant (i.e., there is at most one proof of $x \in U=$ true), this ensures that $\left|\left(\left.G\right|_{U}\right)\right|=|\Sigma x: G . x \in U|=|U|$. This is a standard technique used pervasively in the mathematical components library. 
The mathematical components library includes a predicate and a function

$$
\text { path }: \forall(T: \text { Type }) \text {. rel } T \rightarrow T \rightarrow \text { seq } T \rightarrow \mathbb{B} \quad \text { last }: \forall(T: \text { Type }) . T \rightarrow \text { seq } T \rightarrow T
$$

such that path ex holds if the list $x:: q$ represents a path in the relation $e$, and last $x q$ returns the last element of $x:: q$. The functions path and last account for the nonemptiness of paths though the use of two arguments: the first vertex $x$ and the (possibly empty) list of remaining vertices $q$.

Thus, the notion of an $x y$-path (in some fixed digraph $G$ ) can be formalized as a predicate on lists:

$$
\text { pathp } x \text { y } p:=\text { path }(\operatorname{edge} G) x p \wedge \text { last } x p=y
$$

However, this is cumbersome to use for several reasons: First it leads to many different assumptions, i.e., both $p: \operatorname{seq} G$ and pathp $x y p$ for a single $x y$-path. This is inconvenient since a single lemma can easily involve six or more different paths, thus cluttering the context. Second, " $u$ is a vertex of the $x y$-path $p$ " would be written as $u \in x:: p$, i.e., requiring explicit mention of the first vertex. Lastly and most importantly, the asymmetric definition gets in the way of symmetry reasoning by path reversal (in simple graphs). For instance, consider some $p$ satisfying pathp $x$ y $p$. Then the corresponding $p^{\prime}$ satisfying pathp $y x p^{\prime}$ is obtained by removing the last element of $x:: p$ and reversing the result. In particular $p^{\prime}$ cannot be computed from $p$ alone.

We solve these problems by packaging the predicate pathp into an indexed family of types:

Record Path $x y:=\{$ pval : seq G ; pvalP : pathp $x$ y pval $\}$

This allows us to endow paths with their own membership operation taking care of the fact that the list representing a path does not contain the first vertex. Moreover, it abstracts from the asymmetry in the definition of the path predicate, easing symmetry reasoning. In the following we write $x \rightsquigarrow y$ for the type Path $x y$.

Indexing paths by their endpoints also allows us to define a dependently typed concatenation function "\#" for paths, i.e., for $\pi_{1}: x \rightsquigarrow y$ and $\pi_{2}: y \rightsquigarrow z$ we have $\pi_{1}+\pi_{2}: x \rightsquigarrow z$ as one would expect. This concatenation function turned out to be more convenient in our setting than the one defined in the mathematical components library: the side condition that paths can be concatenated is ensured by the typing discipline. We prove various trivialities about paths (e.g., $x \in \pi$ whenever $\pi: x \rightsquigarrow y$ ) that are then added to the hint databases of standard automation tactics. We also prove a number of lemmas for splitting paths or transferring paths between graphs. For instance, the lemma below, which allows splitting paths at the first vertex satisfying some criterion, is used more than twenty times throughout the development.

Lemma 3.4 Let $G$ be a digraph, $x, y: G, A: 2^{G}$, and $\pi: x \rightsquigarrow y$ such that $\pi \cap A \neq \emptyset$. Then there exist $z: G$ and $\pi_{1}: x \rightsquigarrow z$ and $\pi_{2}: z \rightsquigarrow y$ such that $\pi=\pi_{1}+\pi_{2}$ and $\pi_{1} \cap A=\{z\}$.

Note that a path is always a path in some specific graph, i.e., if $x, y: G$ then there is an implicit $G$ in the type $x \rightsquigarrow y$. On the other hand, a sequence of vertices can give rise to paths in different graphs, e.g., if $\pi: x \rightsquigarrow y$ is a path in $G$, then the underlying sequence of vertices uniquely determines a path in $G+u v$ (which has the same type of vertices). For this reason, we introduce a function, seq_of : $\forall G(x y: G) . x \rightsquigarrow y \rightarrow \operatorname{seq} G$ returning the underlying sequence of vertices. 
Lemma 3.5 Let $G$ be a digraph, let $x, y, u, v: G$, and let $\pi: x \rightsquigarrow y$ be a path in $G$. Then there exists path $\rho: x \rightsquigarrow y$ in $G+$ uv such that seq_of $\rho=$ seq_of $\pi$.

The lemma above exploits that adding an edge does not change the type of vertices. In the case of induced subgraphs, where the type of vertices does change, the corresponding lemma takes a slightly different form. Writing $\left.L_{-}\right\rfloor$for the natural injection from an induced subgraph to the full graph, we have:

Lemma 3.6 Let $G$ be a digraph, let $U: 2^{G}$ and let $\pi: x \rightsquigarrow y$ be a path in $\left.G\right|_{U}$. Then there exists a path $\rho:\lfloor x\rfloor \rightsquigarrow\lfloor y\rfloor$ such that seq_of $\rho=\operatorname{map}\left\lfloor_{-}\right\rfloor$(seq_of $\pi$ ).

The preceding lemmas can be seen as algorithmic constructions on paths. We follow the general design of the mathematical components library and prove these lemmas as propositions corresponding to the wording given (i.e., using existential quantification), allowing us to rely on existential statements from the library.

We remark that we are dealing almost exclusively with decidable properties, and for all the properties we define (e.g., connectedness of sets), we provide a boolean decider and use it when necessary (e.g., in the definition of finite sets or to justify case distinctions). Hence, if one needs to obtain a path in a computational context (i.e., when constructing inhabitants of types that are not propositions) one can use the constructive choice operator to obtain a concrete witness; this is almost never necessary.

Paths give rise to a number of auxiliary notions. One path-based notion that is used pervasively throughout the development is that of a connected set.

Definition 3.7 Let $G$ be a simple graph and let $A, B: 2^{G}$.

- $A$ and $B$ are neighboring if there exist vertices $a \in A$ and $b \in B$, such that $a-b$.

- $A$ is connected if for every $x, y \in A$ there exists an $x y$-path contained in $A$.

- The graph $G$ is connected if the full set of vertices is connected.

We prove a number of lemmas allowing us to establish connectedness of sets. In addition to a number of trivial lemmas (e.g., $\{x, y\}$ is connected whenever $x-y$ ) we also prove closure properties such as:

Lemma 3.8 Let $G$ be a simple graph and let $A: 2^{G}$ and $B: 2^{G}$ be connected.

1. If $A \cap B \neq \emptyset$, then $A \cup B$ is connected.

2. If $A$ and $B$ are neighboring, then $A \cup B$ is connected.

The purpose of the auxiliary notion of neighboring sets is to avoid having to explicitly mention edges in certain situations; we will use it extensively in Sections 6 and 7 .

\section{Connectivity}

Before we turn to the proof of the excluded minor characterization of treewidthtwo graphs, we need a few results of about graph connectivity. The main result of this section is Menger's Theorem [24]. In addition, we establish a number of well-known corollaries of Menger's Theorem and some related results we need in subsequent sections.

Informally, Menger's Theorem states that if one needs to remove at least $n$ vertices to disconnect two sets of vertices $A$ and $B$ of some graph, then there exist $n$ pairwise disjoint paths from $A$ to $B$. The formal statement makes use of the following definition: 
Definition 4.1 Let $G$ be a digraph and $A, B: 2^{G}$. A set $S: 2^{G}$ is an $A B$-separator if every path starting in $A$ and ending in $B$ contains a vertex from $S$. An $A B$ connector is a collection of pairwise disjoint irredundant paths such that every path starts at a vertex in $A$, ends at a vertex in $B$, and has no other vertices in common with either $A$ or $B$.

Menger's Theorem states that the minimum size for $A B$-separators is also the maximum size for $A B$-connectors. Since every $A B$-separator must contain at least one vertex from every path of every $A B$-connector, every $A B$-separator must be at least as large as the largest $A B$-connector. The nontrivial part of the theorem is to show the existence of an $A B$-connector of size $n$ if every $A B$-separator has size at least $n$.

In Coq, there is a complication when defining the notion of connector: indexing the type of paths by the first and last vertex on the path means that in order to form a collection of paths with different endpoints we need to abstract from these endpoints. Thus we define a type of $G$-paths and projection functions yielding respectively the first vertex, the last vertex, and the encapsulated path:

$$
\begin{aligned}
G \text {-path } & :=\Sigma(x, y): G \times G \cdot x \rightsquigarrow y \\
\text { fst }\langle\langle x, y\rangle, \pi\rangle & :=x \\
\text { Ist }\langle\langle x, y\rangle, \pi\rangle & :=y \\
\text { pth }\langle\langle x, y\rangle, \pi\rangle & :=\pi
\end{aligned}
$$

Note that pth : $\forall \pi: G$-path. fst $\pi \rightsquigarrow$ Ist $\pi$, i.e., the return type of pth $\pi$ depends on the value of $\pi$. This is mainly useful in combination with predicates that are parametric in the index-vertices (e.g., irred: $\forall x y: G . x \rightsquigarrow y \rightarrow \mathbb{B}$ ) or when viewing paths as sets.

With this in place, $A B$-connectors of size $n$ can be defined as predicates on functions $X: I_{n} \rightarrow G$-path. Writing $X_{i}$ for the application $X i$, we have:

$$
\begin{aligned}
A B \text {-connector } X & :=\forall i: I_{n} . \text { irred } X_{i} \\
& \wedge \forall i: I_{n} . X_{i} \cap A=\left\{\text { fst } X_{i}\right\} \\
& \wedge \forall i: I_{n} . X_{i} \cap B=\left\{\text { lst } X_{i}\right\} \\
& \wedge \forall i j: I_{n} . i \neq j \rightarrow X_{i} \cap X_{j}=\emptyset
\end{aligned}
$$

Before we can prove Menger's Theorem, we need two technical lemmas that respectively allow the extension of a connector with a single edge and the concatenation of two connectors.

Lemma 4.2 Let $G$ be a digraph, $A$ and $B$ sets of vertices of $G, j: I_{n}$ and $X: I_{n} \rightarrow$ $G$-path an $A B$-connector. If $x \notin \bigcup_{i} X_{i}$, fst $\left(X_{j}\right)=y, x-y$ and $x \notin B$, then there exists an $(\{x\} \cup A \backslash\{y\}) B$-connector of size $n$.

Proof Follows by prepending $x$ to $X_{j}$.

Lemma 4.3 Let $G$ be a digraph, $A$ and $B$ sets of vertices of $G$, and $P$ an $A B$-separator with $|P|=n$. Further let $X: I_{n} \rightarrow G$-path an $A P$-connector and $Y: I_{n} \rightarrow G$-path $a$ $P B$-connector. Then there exists an AB-connector of size $n$. 


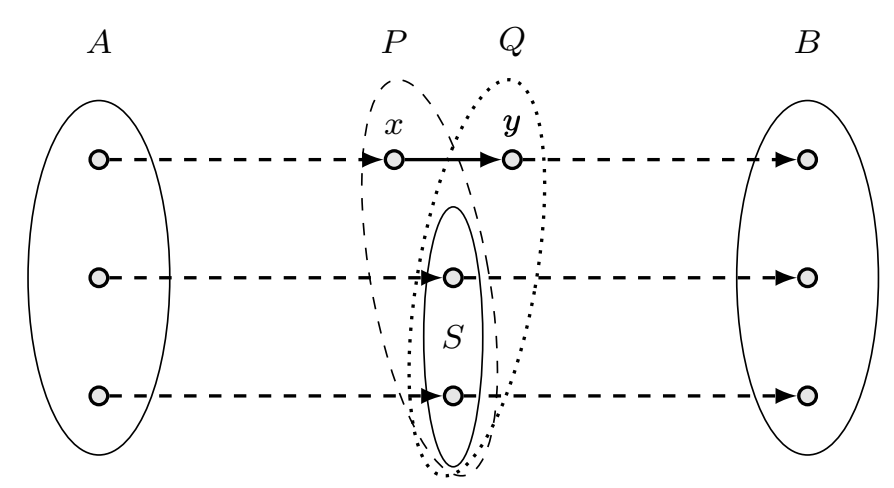

Fig. 1: Objects occuring the the proof of Menger's Theorem

Proof Since all $X_{i}$ (as well as all $Y_{i}$ ) are mutually disjoint and each $X_{i}$ and $Y_{j}$ contains a single vertex from $P$, there exists a bijection $m: I_{n} \rightarrow I_{n}$ such that that Ist $\left(X_{i}\right)=\mathrm{fst}\left(Y_{m(i)}\right)$. Since $P$ is an $A B$-separator, $X_{i}$ and $Y_{j}$ (for any $i$ and $j$ ) share at most at a single vertex of $P$ (in this case $j=m(i)$ ). Thus, the function $Z_{i}:=X_{i}+Y_{m(i)}$ is a connector as required.

We sketch the argument that $Z_{i} \cap A=\left\{\right.$ fst $\left.\left(Z_{i}\right)\right\}$. Assume $X_{i}$ is an $x y$-path and $Y_{m(i)}$ is a $y z$-path. The inclusion from right to left is trivial, as is showing that $X_{i} \cap A \subseteq\left\{\right.$ fst $\left.\left(Z_{i}\right)\right\}$. So assume some $u \in Y_{m(i)} \cap A$. It suffices to show $u=y$ for then $u \in X_{i}$. This follows since the $u z$-part of $Y_{m(i)}$ is an $A B$-path and therefore must contain a vertex $v \in P$. But $y$ is the only vertex in $P \cap Y_{m(i)}$, so $v=y=u$ since $Y_{m(i)}$ is irredundant.

The use of "\#" in the definition of $Z_{i}$ is a slight abuse of notation since so far we only defined concatenation for vertex-indexed paths with matching ends. In the case of $G$-paths, we extend the usual concatenation function with a check ensuring that the two paths do compose and only perform the concatenation in this case (using the equality generated by the check to align the types). In the above construction, this is always the case and we can establish this once and for all. Thus, the verification that $Z_{i}$ is indeed a connector closely follows the provided proof sketch.

Theorem 4.4 (Menger's Theorem) Let $G$ be a digraph and let $A, B$ be sets of vertices of $G$ such that every $A B$-separator has at least size $n$. Then there exists an $A B$-connector of size at least $n$.

Proof By induction on $|\{(x, y): G \times G \mid x-y\}|$. If $G$ has no edges, then $A \cap B$ (seen as a collection of single-vertex paths) is a sufficiently large $A B$-connector. Hence, we can assume there are vertices $x, y: G$ such that $x-y$. Let $G^{\prime}:=G-x y$. Without loss of generality, $G^{\prime}$ has an $A B$-separator $S$ with $|S|<n$. [Otherwise, we obtain the required $A B$-connector by induction.] Let $P:=S \cup\{x\}$ and $Q:=S \cup\{y\}$. Both $P$ and $Q$ are $A B$-separators of $G$. [Assume there was some $A B$-path $\pi$ in $G$ avoiding $x$ or $y$. Then $\pi$ cannot use the $x y$-edge, hence $\pi$ is a $G^{\prime}$-path and visits $S$.] Thus, $n=|P|=|Q|=|S|+1$, i.e., $x, y \notin S$ and the situation looks as depicted in Figure 1. Now, every $A P$-separator (or $Q B$-separator) of $G^{\prime}$ is an $A B$-separator of $G$. [To see this, let $T$ be an $A P$-separator of $G^{\prime}$ and assume $\pi$ is an $A B$-path in $G$ avoiding $T$. Then $\pi$ visits the $A B$-separator $P$, and therefore must use the $x y$-edge. 
Splitting $\pi$ at $x$ yields an $A P$-path in $G^{\prime}$ avoiding $T$. Contradiction.] Thus, every $A P$-separator (or $Q B$-separator) of $G^{\prime}$ has size at least $n$. By induction hypothesis, we obtain an $A P$-connector $X$ and a $Q B$-connector such that $|X|=|Y|=n$. The required $A B$-connector is then obtained using Lemmas 4.2 and 4.3 .

The above proof is almost exactly the proof given by Göring [18], we merely add some additional elaborations (i.e., the sentences enclosed in "[...]" and the two lemmas). We remark that Menger's theorem is a special case of the max-flow mincut theorem. The latter was recently formalized in Isabelle/HOL together with the Edmonds-Karp algorithm for computing maximum flows [22]. We restrict ourselves to proving Menger's theorem because Menger's theorem is sufficient for our purposes and allows for a much simpler proof than the max-flow min-cut theorem.

Several important theorems (e.g., Hall's Marriage Theorem and Kőnig's Theorem) can be obtained as simple consequences of Menger's Theorem.

Hall's Marriage Theorem. We first prove a variant of Hall's Marriage Theorem for bipartite directed graphs that follows naturally from Menger's Theorem for directed graphs. As a second step, we derive the usual formulation of Hall's Marriage Theorem for bipartite simple graphs.

Definition 4.5 Let $G$ be a digraph. We define $\mathrm{N}(A):=\{y \in \bar{A} \mid x-y\}$ (with $\bar{A}$ being the complement of $A$ in $G$ ) to be the neighborhood of $A$. A bipartition of $G$ is set $A$ of vertices of $G$, such that for every edge of $G$ exactly one of the ends is in $A$ (i.e., $(x \in A) \oplus(y \in A)$ whenever $x-y)$. A directed matching of $G$ is a set $M$ of directed edges (i.e., a set of pairs of vertices $(x, y)$ such that $x-y$ for all pairs) in $G$ such that no two edges in $M$ share a vertex. If $G$ is simple, an (undirected) matching of $G$ is a set $M$ of edges in $G$ (i.e., a set of sets $\{x, y\}$ such that $x-y)$ that is pairwise disjoint.

An $A \bar{A}$-connector in a graph with bipartition $\mathrm{A}$ is essentially a matching.

Lemma 4.6 Let $G$ be a digraph with bipartition $A$ and let $X: I_{n} \rightarrow G$-path be an $A \bar{A}$-connector. Then $\left\{\left(\right.\right.$ fst $X_{i}$, Ist $\left.\left.X_{i}\right) \mid i: I_{n}\right\}$ is a directed matching of size $n$.

Corollary 4.7 Let $G$ be a digraph with bipartition $A$ such that $|\mathrm{N}(S)| \geq|S|$ for all $A \subseteq$ $S$. Then there exists a directed matching $M$ (of $G$ ) such that $A=\{x \mid \exists y .(x, y) \in M\}$

Proof By Lemma 4.6, it suffices to show that every $A \bar{A}$-separator has size at least $|A|$ and obtain an $A \bar{A}$-connector of size $|A|$ using Menger's Theorem. Let $S$ be an $A \bar{A}$-separator. Then $|A|=|A \cap S|+|A \backslash S| \leq|A \cap S|+|\mathrm{N}(A \backslash S)| \leq|S|$. The first inequality holds by assumption, the second inequality holds since the two sets are disjoint and (because $S$ is an $A \bar{A}$-separator) also included in $S$.

For every directed matching $M$ of some graph $G$, the set $\{\{x, y\} \mid(x, y) \in M\}$ is a matching of size $|M|$ covering the same vertices as $M$. Thus, the usual formulation of Hall's Marriage theorem follows immediately with Corollary 4.7.

Theorem 4.8(Hall) Let $G$ be a simple graph with bipartition $A$ such that $|\mathrm{N}(S)| \geq|S|$ for all $A \subseteq S$. Then there exists a matching $M$ (of $G$ ) such that $A \subseteq \bigcup M$. 
König's Theorem The theorem states that in bipartite graphs the size of a minimum vertex cover and a maximum matching are the same. This is is another well-known and widely-used consequence of Menger's Theorem.

Definition 4.9 Let $G$ be a simple graph. A set $V$ of vertices of $G$ is called a vertex cover if every edge in $G$ has at least one end in $V$. A vertex cover is minimum if no vertex cover has fewer vertices. A matching of $G$ is maximum if no matching has more edges.

Lemma 4.10 Let $G$ be a simple graph, let $V$ be a vertex cover of $G$ and let $M$ be a matching of $G$. Then $|M| \leq|V|$. Moreover, if $|M|=|V|$ we also have $V \subseteq \bigcup M$.

Proof The edges in $M$ are pairwise disjoint and each share a vertex with $V$. Thus there exists an injective function $f$ from $M$ to $V$. This yields $|M| \leq|V|$. If $|M|=|V|$, then $f$ must also be surjective and we obtain $V \subseteq \bigcup M$.

Corollary 4.11 Let $G$ be bipartite and let $V$ be a minimum vertex cover. Then there exists a matching of $G$ with $|V| \leq|M|$.

Proof Let $A$ be a bipartition of $G$. It is easy to see that every $A \bar{A}$-separator is also a vertex cover. Thus, the claim follows with Menger's Theorem and Lemma 4.6.

Theorem 4.12 (König) Let $G$ be bipartite, let $V$ a minimum vertex cover, and let $M$ be a maximum matching. Then $|V|=|M|$.

Proof We have $|V| \geq|M|$ by Lemma 4.10. Since $M$ is maximum, it suffices to obtain some matching $M^{\prime}$ with $|V| \leq\left|M^{\prime}\right|$. Thus, the claim follows with Corollary 4.11.

Independent Paths In the sequel, we will only make use of a variant of Menger's Theorem establishing the existence of $n$ independent paths between a pair of vertices $x$ and $y$ provided one needs to remove at least $n$ vertices to disconnect $x$ and $y$.

Definition 4.13 Let $x, y$ be vertices of some digraph $G$. Two irredundant $x y$-paths $\pi_{1}$ and $\pi_{2}$ are independent if $\pi_{1} \cap \pi_{2}=\{x, y\}$. A set of vertices $S$ separates $x$ and $y$ if $\{x, y\} \cap S=\emptyset$ and every $x y$-path contains a vertex from $S$.

Note that " $S$ separates $x$ and $y$ " is a stronger statement than " $S$ is a $\{x\}\{y\}$ separator". In particular, $\{x\}$ is always an $\{x\}\{y\}$-separator and never separates $x$ and $y$.

Corollary 4.14 Let $G$ be a digraph, and let $x, y: G$ such that $x \neq y$ and there is no $x y$-edge. If $n \leq|S|$ for every set $S$ separating $x$ and $y$, then there exist $n$ irredundant and pairwise independent $x y$-paths.

Proof Let $G^{\prime}:=\left.G\right|_{\{x, y\}}$ be the subgraph of $G$ induced by the complement of $\{x, y\}$. Let $A:=\left\{z: G^{\prime} \mid x-z\right\}$ and $B:=\left\{z: G^{\prime} \mid z-y\right\}$. Then every $A B$-separator of $G^{\prime}$ also separates $x$ and $y$ in $G$ and therefore has size at least $n$. By Menger's Theorem, we obtain an $A B$-connector $X$ of size $n$. Adding $x$ at the start and $y$ at the end of every path in $X$ yields $n$ independent $x y$-paths. 

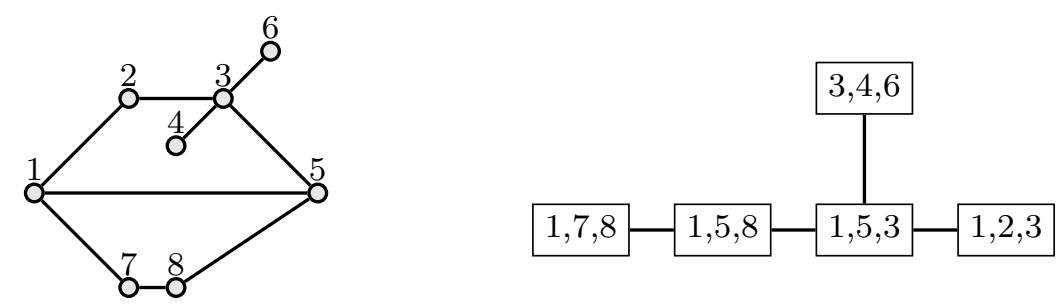

Fig. 2: A graph of treewidth two (left) with tree decomposition (right)

We also prove a variant of the corollary above establishing the existence of edgedisjoint paths in multigraphs (as introduced in Section 8). For additional detail, we refer to the accompanying Coq development [9].

Separators and separating sets not only facilitate the construction of independent paths, they are also a useful tool for decomposing graphs, and we will make use of this in the upcoming sections.

Definition 4.15 (Separators and Connectivity) Let $G$ be a simple graph. A (vertex) separator is a set $S$ disconnecting $G$, i.e., separating two distinct vertices of $G$. The graph $G$ is $k$-connected if $|G|>k$ and every separator has at least $k$ vertices.

Note that the notion of a (vertex) separator is distinct from the $A B$-separators used in the proof of Menger's theorem. Also note that removing a separator may introduce an arbitrary number of disconnected components. However, we sometimes want to view a separator as separating a graph into exactly two (not necessarily connected) components. This motivates the notion of separation.

Definition 4.16 (Separation) Let $G=\langle V, R\rangle$ be a simple graph. A pair of sets $\left(V_{1}, V_{2}\right)$ is a separation (of $G$ ) if $V_{1} \cup V_{2}=V$ and $\overline{V_{2}}$ and $\overline{V_{1}}$ are not neighboring. ${ }^{3}$ A separation is proper if both $\overline{V_{1}}$ and $\overline{V_{2}}$ are nonempty. The order of a separation is the size of $V_{1} \cap V_{2}$. A minimal separation is a separation whose order is minimal.

The main difference between a separator and a proper separation is that the latter specifies for every vertex which "side" of the separator the vertex is on.

Lemma 4.17 1. If $\left(V_{1}, V_{2}\right)$ is a separation, then $V_{1} \cap V_{2}$ separates all elements of $\overline{V_{1}}$ from all elements of $\overline{V_{2}}$. Thus, $V_{1} \cap V_{2}$ is a separator whenever $\left(V_{1}, V_{2}\right)$ is proper.

2. For every separator $S$, there exists a proper separation $\left(V_{1}, V_{2}\right)$, with $V_{1} \cap V_{2}=S$.

\section{Treewidth}

We now define the notions of treewidth and minors. Both notions appear in the literature with slight (but equivalent) variations. We choose variants that yield reasonable proof principles.

Definition 5.1 A forest is a simple graph where there is at most one irredundant path between any two nodes (i.e., any two irredundant $x y$-paths are equal).

${ }^{3}$ Note that $\overline{V_{2}}=V_{1} \backslash V_{2}$ if $V_{1} \cup V_{2}=V$. 
Definition 5.2 A tree decomposition of a simple graph $G$ is a forest $F$ together with a function $B: F \rightarrow 2^{G}$ such that:

T1. for every vertex $x: G$, there exists some $t: F$, such that $x \in B(t)$.

T2. for every $x: G$, the set $\{t: F \mid x \in B(t)\}$ is connected in $F$;

T3. if $x-y$, then there exists a node $t$, such that $\{x, y\} \subseteq B(t)$;

The width of a tree decomposition is the size of the largest set $B(t)$ minus one; the treewidth of a graph is the minimal width of a tree decomposition.

An example of a graph together with a tree decomposition certifying that the graph has treewidth at most two is given in Figure 2.

Remark 5.3 The minus one in the definition of treewidth is there solely to ensure that trees have treewidth one. In the formalization we take the width so be the size of the largest bag (without substracting one) and adapt the statements accordingly. Moreover, we only formalize the proposition "has treewidth at most $k$ ", which asserts the existence of a tree decomposition of width at most $k$. We do not define a function computing the treewidth of a given graph, because this is not required for any of the results presented here.

Note that we define the notion of tree decomposition using forests rather than trees as is done for instance in [8]. The two notions are equivalent since every forest can be turned into a tree by connecting arbitrary nodes of disconnected trees. Using forests rather than trees has the advantage that tree decompositions for the disjoint union of two graphs $G$ and $H$ can be obtained as the disjoint union of tree decompositions for $G$ and $H$.

Definition 5.4 Let $G$ and $H$ be simple graphs. We write $G+H$ for the disjoint union of $G$ and $H$ (i.e., the graph with vertices $\operatorname{inl} x$ for $x: G$ and inr $y$ for $y: H$ such that inl $x-\operatorname{inl} y$ iff $x-y$ in $G$ and likewise for $H$ ).

Lemma 5.5 Let $G_{1}$ and $G_{2}$ be simple graphs, $T_{1}$ and $T_{2}$ forests, $B_{1}: T_{1} \rightarrow 2^{G_{1}}$ a tree decomposition of $G_{1}$, and $B_{2}: T_{2} \rightarrow 2^{G_{2}}$ a tree decomposition of $G_{2}$. Then

$$
\lambda u: T_{1}+T_{2} \cdot \begin{cases}B_{1}(x) & u=\operatorname{inl} x \\ B_{2}(x) & u=\operatorname{inr} x\end{cases}
$$

is a tree decomposition of $G_{1}+G_{2}$.

One crucial property of tree decompositions is that a tree decomposition of a graph $G$ must contain every clique in $G$ in one of the bags.

Definition 5.6 Let $G$ be a simple graph and let $A: 2^{G}$. $A$ is a clique if $x-y$ whenever $\{x, y\} \subseteq A$ and $x \neq y$.

Theorem 5.7 Let $T$ be a (nonempty) forest and let $B: T \rightarrow 2^{G}$ be a tree decomposition of $G$. Then every clique of $G$ is contained in $B(t)$ for some $t: T$.

Proof We prove by induction on $n$ that every clique $S$ with $|S| \leq n$ is contained in $B(t)$ for some $t$. If $|S| \leq 1$ this follows from (T1). Thus, we can assume $\left\{v_{0}, v\right\} \subseteq S$ with $v_{0} \neq v$. We define

$$
S_{0}:=S \backslash\{v\} \quad T_{0}:=\left\{t: T \mid S_{0} \subseteq B(t)\right\}
$$


By the induction hypothesis, $T_{0}$ is nonempty. Moreover, we can assume $v \notin B(t)$ for all $t \in T_{0}$. Since $S$ is a clique, every set $\{x, y\} \subseteq S$ is contained in $B(t)$ for some $t: T$ (T3). Let $c$ be such that $\left\{v, v_{0}\right\} \subseteq B(c)$ and let $C$ be the component of $c$ in $T \backslash T_{0}$, i.e.:

$$
C:=\left\{t: T \mid \exists \pi: c \rightsquigarrow t . \pi \cap T_{0}=\emptyset\right\}
$$

Then $C$ is connected, disjoint from $T_{0}$ and contains $c$. Since $v_{0}$ occurs both in $T_{0}$ and in $C$, there exist $t_{0} \in T_{0}$ and $c_{0} \in C$ such that $t_{0}-c_{0}$ (T2) and, since $T$ is a forest, every path starting in $C$ and ending in $T_{0}$ must use this edge. We obtain a contradiction by showing $c_{0} \in T_{0}$.

Fix some $u \in S_{0}$; it suffices to show $u \in B\left(c_{0}\right)$. We have $u \in B\left(t_{0}\right)$. By (T3) we also have $\{u, v\} \subseteq B\left(c_{u}\right)$ for some $c_{u}$. Since $v \subseteq B(c) \cap B\left(c_{u}\right)$ the unique irredundant $c c_{u}$-path avoids $T_{0}$ (T2). Thus $c_{u} \in C$ and therefore $u \in B\left(c_{0}\right)$ since the unique $t_{0} c_{u}$-path must go through $c_{0}$ and (again by T2) every bag along the way must contain $u$.

Theorem 5.7 provides a useful criterion to establish lower bounds for the treewidth of a given graph (e.g., $\mathrm{K}_{n+1}$, the complete graph with $n+1$ vertices, has treewidth at least $n$ ). Moreover, it provides a way to obtain a tree decomposition for a graph from tree decompositions of a separation whose shared part is a clique.

Lemma 5.8 Let $G$ be a graph and let $\left(V_{1}, V_{2}\right)$ be a separation of $G$ such that $V_{1} \cap V_{2}$ is a clique. Further let $\left(T_{i}, B_{i}\right)$ be a tree decomposition of $\left.G\right|_{V_{i}}$ for $i \in\{1,2\}$. Then there exists a tree decomposition of $G$ of width $\max \left(\operatorname{width}\left(B_{1}\right), \operatorname{width}\left(B_{2}\right)\right)$.

Proof Since $V_{1} \cap V_{2}$ is a clique, there exist nodes $t_{i}: T_{i}$ such that $V_{1} \cap V_{2} \subseteq B_{i}\left(t_{i}\right)$ for $i \in\{1,2\}$ (Theorem 5.7). We obtain a tree decomposition of $G$ by taking the disjoint union of the tree decompositions of $\left.G\right|_{V_{1}}$ and $\left.G\right|_{V_{2}}$ and then adding a $t_{1} t_{2^{-}}$ edge.

Lemma 5.9 Forests have treewidth at most one.

Proof Let $F$ be a forest. We proceed by induction on $|F|$. If $|F| \leq 2$, the claim is trivial. If $3 \leq|F|$, it is straightforward to see that $|F|$ has a separator $S$ with $|S| \leq 1$. We obtain a proper separation $\left(V_{1}, V_{2}\right)$ of $F$ with $S=V_{1} \cap V_{2}$ (Lemma 4.17). By induction hypothesis, we obtain tree decompositions of width at most 1 for $\left.F\right|_{V_{1}}$ and $\left.F\right|_{V_{2}}$. Thus, we obtain the required tree decomposition by Lemma 5.8.

Note that Lemma 5.8 uniformly deals with the cases of disconnected components and components separated by a single vertex.

\section{Minors}

The minors of a graph $G$ are customarily defined to be those graphs that can be obtained by a series of the following operations: remove a vertex, remove an edge, or contract an edge. Instead, we use a monolithic definition in terms of functions to sets inspired by [8].

Definition 6.1 Let $G$ and $H$ be simple graphs. A function $\phi: H \rightarrow 2^{G}$ is called a minor map if:

M1. $\phi(x)$ is nonempty and connected for all $x: H$, 

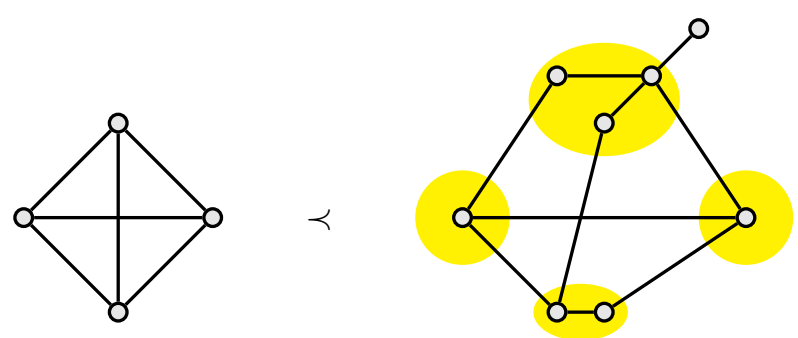

Fig. 3: Exhibiting $\mathrm{K}_{4}$ as a minor

M2. $\phi(x) \cap \phi(y)=\emptyset$ whenever $x \neq y$ for all $x, y: H$.

M3. $\phi(x)$ neighbors $\phi(y)$ for all $x, y: H$ such that $x-y$.

$H$ is a minor of $G$, written $H \prec G$ if there exists a minor map $\phi: H \rightarrow 2^{G}$.

Intuitively, for every vertex $x: H, \phi(x)$ is the set of vertices being collapsed to $x$ by contracting edges in $\phi(x)$. Consequently we will refer to $\phi(x)$ as the inflation of $x$.

In [10], we employed an equivalent definition using functions of type $G \rightarrow H_{\perp}$ instead of $H \rightarrow 2^{G}$ and expressing the conditions on the preimages. For the results in this paper, we found Definition 6.1 more convenient; the Coq development contains both definitions and establishes their equivalence.

As an example, consider the graph obtained by adding an edge between vertices 4 and 7 of the graph in Figure 2. This graph (depicted on the right of Figure 3) admits $\mathrm{K}_{4}$ as minor by collapsing the circled sets of vertices. Note that there are in general many ways to contract a connected set to a single vertex and our definition abstracts from these unimportant variations.

Lemma 6.2 If $\phi: G \rightarrow 2^{H}$ and $\psi: H \rightarrow 2^{I}$ are minor maps, then $\lambda x$. $\bigcup_{y \in \phi(x)} \psi(y)$ is a minor map of type $G \rightarrow 2^{H}$.

As a consequence of the lemma above, we obtain that $\prec$ is transitive. Moreover, we have that the class of graphs with treewidth below a certain threshold is closed under taking minors.

Lemma 6.3 If $H \prec G$, then the treewidth of $H$ is at most the treewidth of $G$.

Proof Let $(T, B)$ be a tree decomposition of $G$ and let $\phi: H \rightarrow 2^{G}$ be a minor map. Then $D(t):=\{x: H \mid \phi(x) \cap B(t) \neq \emptyset\}$ is a tree decomposition of $H$. Moreover, $\phi(x)$ and $\phi(y)$ are disjoint whenever $x \neq y$, so $|D(t)| \leq|B(t)|$.

One of our main results is a formal proof that the $\mathrm{K}_{4}$-free graphs are precisely those graphs that have tree decompositions of width at most two. One direction, showing that a graph cannot both have a tree decompositon of width at most two and $\mathrm{K}_{4}$ as a minor, is a straightforward consequence of Theorem 5.7 and Lemma 6.3. More generally, we have:

Definition 6.4 We write $\mathrm{K}_{n}$ for the complete graph with $n$ vertices. A graph $G$ is called $\mathrm{K}_{n}$-free, if $\mathrm{K}_{n} \nprec G$.

Proposition 6.5 Let $k \geq 0$. If $G$ has treewidth at most $k$, then $\mathrm{K}_{k+2} \nprec G$. 


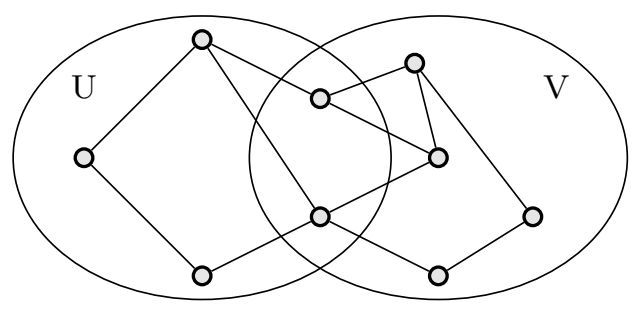

Fig. 4: Separation of a graph with a smallest separator of size two.

For $k \leq 2$, Proposition 6.5 is actually an equivalence. For $k=0$, this is trivial: if $K_{2} \nprec G$ then $G$ is edgeless and we can put every vertex in a bag of its own in a forest of isolated vertices. We prove the case for $k=1$ below and we prove the case for $k=2$ in the next section. To facilitate the proofs, we introduce an auxiliary notion. If $\pi$ is an $x y$-path, we write $[\pi]$ for the set $\pi \backslash\{x, y\}$. Note that if $\pi$ is irredundant, then $[\pi]$ is connected; if $[\pi]$ is also nonempty, then $[\pi]$ neighbors any set containing either $x$ or $y$.

Theorem 6.6 The following are equivalent:

1. $G$ is a forest

2. G has treewidth at most one.

3. $\mathrm{K}_{3}$ is not a minor of $G$.

Proof We already showed (1) $\Rightarrow(2)$ (Lemma 5.9) and (2) $\Rightarrow(3)$ (Proposition 6.5). Since being a forest is a decidable property, we can show $(3) \Rightarrow(1)$ by contraposition. Assume $G$ is not a forest, i.e., assume vertices $x, y: G$ and irredundant paths $\pi_{1}, \pi_{2}: x \rightsquigarrow y$ such that $\pi_{1} \neq \pi_{2}$. Without loss of generality, we can assume $\left[\pi_{1}\right] \cap\left[\pi_{2}\right]=\emptyset$ and $\left[\pi_{1}\right] \neq \emptyset$. Mapping the three vertices of $\mathrm{K}_{3}$ to $\{x\},\left[\pi_{1}\right]$, and $\left[\pi_{2}\right] \cup\{y\}$ yields $\mathrm{K}_{3} \prec G$ as required.

\section{Tree Decompositions for $\mathbf{K}_{4}$-free Graphs}

We now prove that every $\mathrm{K}_{4}$-free graph has a tree decomposition of width at most two. For this, we need to construct an object (a low-width tree decomposition) from the knowledge that a certain substructure (the minor $\mathrm{K}_{4}$ ) does not occur.

The proof we give is inspired by the argument in [8]; it is structured as follows: We first show that every sufficiently large $\mathrm{K}_{4}$-free graph can be covered by two sets $U$ and $V$ whose intersection contains at most two vertices (cf. Figure 4). This part makes use of Menger's Theorem. We then show that if $U \cap V$ does contain two vertices, we can assume without loss of generality that there is an edge between them. This allows us to obtain a tree decomposition of $G$ by composing tree decompositions of the subgraphs $\left.G\right|_{U}$ and $\left.G\right|_{V}$ (Lemma 5.8). Arguing that $G+x y$ is still $\mathrm{K}_{4}$-free in the case where $U \cap V=\{x, y\}$ but $x$ and $y$ are not adjacent (the nontrivial part of the without loss generality argument) requires analyzing a hypothetical minor map exhibiting $\mathrm{K}_{4}$ as a minor of $G+x y$ and showing that this would yield $\mathrm{K}_{4} \prec G$. This is the most technical part of the proof.

Lemma 7.1 Every 3-connected graph includes $\mathrm{K}_{4}$ as a minor. 


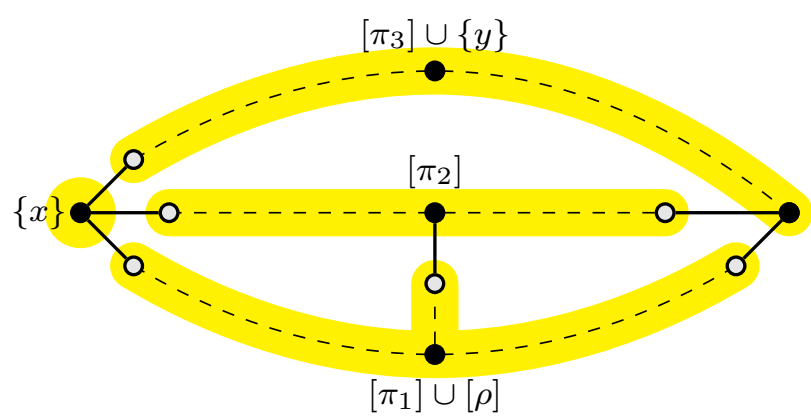

Fig. 5: Constructing $\mathrm{K}_{4}$ from a separator of size three: The filled vertices are distinct, unfilled vertices may coincide with the filled vertices they are connected to by dashed lines.

Proof Let $G$ be 3 -connected. If $G$ is complete, we clearly have $\mathrm{K}_{4} \prec G$. Otherwise let $x$ and $y$ be two vertices such that there is no $x y$-edge. By Corollary 4.14, we obtain three independent $x y$-paths $\pi_{1}, \pi_{2}$ and $\pi_{3}$. Moreover, all these paths have a non-empty interior. Since $\{x, y\}$ is not a separator, there exists a path $\rho$ connecting, without loss of generality, $\left[\pi_{1}\right]$ and $\left[\pi_{2}\right]$ such that $[\rho] \cap\left(\pi_{1} \cup \pi_{2} \cup \pi_{3}\right)=\emptyset$. The minor map mapping the four vertices of $\mathrm{K}_{4}$ to the following four sets establishes $\mathrm{K}_{4}$ as a minor of $G:\{x\},\left[\pi_{1}\right] \cup[\rho],\left[\pi_{2}\right]$, and $\left[\pi_{3}\right] \cup\{y\}$ (cf. Figure 5).

As an immediate consequence of the lemma above we obtain the existence of small separators in $\mathrm{K}_{4}$-free graphs.

Lemma 7.2 Let $G$ be a simple $\mathrm{K}_{4}$-free graph with at least four vertices. Then there exists a smallest separator $S$ of $G$ with $|S| \leq 2$.

In order to use the aforementioned small separators to decompose graphs in such a way that their respective tree decompositions yield a tree decomposition of the full graph, we need to show that if $\{x, y\}$ is a smallest separator, then adding an $x y$-edge preserves $\mathrm{K}_{4}$-freeness. We first establish two auxiliary lemmas.

Lemma 7.3 Let $\mathrm{K}_{4} \prec G$ and let $\left(V_{1}, V_{2}\right)$ be a minimal separation of $G$ such that $V_{1} \cap V_{2}$ is a clique of size at most two. Then there exists a minor map $\phi$ such that either $\phi(x) \subseteq V_{1}$ for all $x: \mathrm{K}_{4}$ or $\phi(x) \subseteq V_{2}$ for all $x: \mathrm{K}_{4}$.

Proof Let $G=\langle V, E\rangle$ and let $\phi: \mathrm{K}_{4} \rightarrow 2^{G}$ be a minor map. We first show that, without loss of generality, we can assume $\phi(i) \cap V_{1} \neq \emptyset$ for all $i$. To see this, consider $i, j$ such that $\phi(i) \cap V_{1}=\emptyset$ and $\phi(j) \cap V_{2}=\emptyset$. If $i=j$, we have a contradiction since $\phi(i)$ is nonempty and $V_{1} \cup V_{2}=V$. Similarly, $i \neq j$ contradicts the assumption that $\overline{V_{1}}$ does not neighbor $\overline{V_{2}}$. Now, since $\left(V_{1}, V_{2}\right)$ is a separation, every $\phi(i)$ containing a vertex from $\overline{V_{1}}$ must also contain a vertex from $S:=V_{1} \cap V_{2}$. Thus, $\phi^{\prime}(i):=\phi(i) \cap V_{1}$ is a minor map as required (using the fact that $S$ is a clique to ensure that the various $\phi(i)$ are connected and neighboring).

Lemma 7.4 If $\left(V_{1}, V_{2}\right)$ is a minimal proper separation, then every vertex $x \in V_{1} \cap V_{2}$ is adjacent to a vertex in $\overline{V_{1}}$ and a vertex in $\overline{V_{2}}$.

The following lemma is the central construction of this section. 
(a)

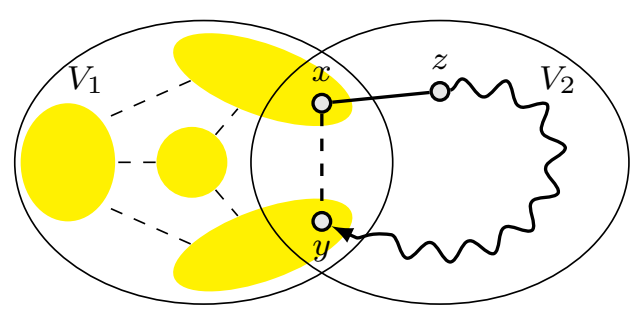

(b)

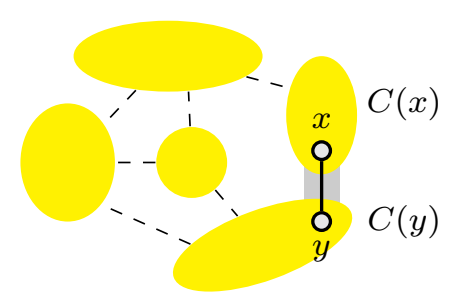

Fig. 6: Possible uses of the $x y$-edge by $\phi: \mathrm{K}_{4} \rightarrow 2^{G+x y}$

Lemma 7.5 Let $G$ be $\mathrm{K}_{4}$-free and let $S=\{x, y\}$ (with $x \neq y$ ) be a smallest separator. Then $G+x y$ is $\mathrm{K}_{4}$-free.

Proof Assume $\mathrm{K}_{4} \prec G+x y$ and let $\phi: \mathrm{K}_{4} \rightarrow 2^{(G+x y)}$ be a minor map. Further, let $\left(V_{1}, V_{2}\right)$ be a proper separation with $V_{1} \cap V_{2}=S=\{x, y\}$. By Lemma 7.3, we can assume, without loss of generality, that $\phi(i) \subseteq V_{1}$ for all $i$. Since $G$ is $\mathrm{K}_{4}$-free, the $x y$-edge must be used in one of two ways: (a) to ensure that $\phi(i)$ and $\phi(j)$ are neighboring (in $G+x y$ ) for some $\phi(i)$ and $\phi(j)$ not neighboring in $G$; or (b) to ensure that $\phi(i)$ is connected (in $G+x y$ ) for some $\phi(i)$ not connected in $G$.

Case (a): Without loss of generality, we have $x \in \phi(i)$ and $y \in \phi(j)$. By Lemma 7.4, there exists a vertex $z \notin V_{1}$ such that $x-z$. Since $\{x\}$ is not a separator, we obtain an irredundant $z y$-path $\pi$ avoiding $x$. Since $S$ is a separator, $\pi \cap V_{1}=\{y\}$. Thus, $\phi[j:=\phi(j) \cup \pi]: \mathrm{K}_{4} \rightarrow G$ is a minor map, contradicting the assumption that $G$ is $\mathrm{K}_{4}$-free (Figure $6(\mathrm{a})$ )

Case (b): Let $C(v)$ be the component of $v$ in $\phi(i)$ (seen as a set in $G$ ). Then $\phi(i)=C(x) \cup C(y)$ and $C(x)$ and $C(y)$ are disjoint. If all $\phi(j)$ for $j \neq i$ are neighboring one of the two components (say $C(x)$ ), then $\phi[j:=C(x)]$ is a minor map not using the $x y$-edge, again contradicting the $\mathrm{K}_{4}$-freeness of $G$. Otherwise, we obtain without loss of generality some $j$ such that $\phi(j)$ neighbors $C(x)$ while $\phi(k)$ neighbors $C(y)$ for $k \notin\{i, j\}$ (Figure 6(b)). Setting $\phi^{\prime}:=\phi[j:=\phi(j) \cup$ $C(x), i:=C(y)$ ] (i.e., moving $C(x)$ from $\phi(i)$ to $\phi(j)$ ) yields a minor map where the $x y$-edge is used to connect $\phi(i)$ and $\phi(j)$, reducing the problem to case A.

Note that the monolithic definition of minor maps (Definition 6.1) allows for a straightforward analysis of how the $x y$-edge must be used by the minor map $\phi$. Also note that the notion of neighboring sets is used pervasively throughout this section, allowing us to avoid having to explicitly exhibit edges in many cases. Putting everything together, we obtain:

Theorem 7.6 Every $\mathrm{K}_{4}$-free graph has a tree decomposition of width at most two.

Proof By induction on $|G|$ for some $\mathrm{K}_{4}$-free graph $G$. If $|G| \leq 3$, the claim is trivial; so assume $4 \leq|G|$. By Lemma 7.2 , we obtain a smallest separator $S$ such that $|S| \leq 2$. We can assume without loss of generality that $S$ is a clique: if $S=\{x, y\}$ but $x+y$, then $G+x y$ is $\mathrm{K}_{4}$-free by Lemma 7.5 , and any tree decomposition for $G+x y$ is also a tree decomposition for $G$. We extend $S$ into a proper separation $\left(V_{1}, V_{2}\right)$ with $S=V_{1} \cap V_{2}$ (Lemma 4.17). By induction hypothesis, we obtain tree decompositions for $\left.G\right|_{V_{1}}$ and $\left.G\right|_{V_{2}}$ of width at most two. Thus, we obtain the desired tree decomposition by Lemma 5.8 . 
Note that the proof of Theorem 7.6 has essentially the same structure as the proof of Lemma 5.9. The main difference is that separators of size at most one are trivially cliques, whereas the case of a separator of size two requires an elaborate "without loss of generality" argument.

\section{Labeled multigraphs}

The initial motivation of this work was the characterization of $\mathrm{K}_{4}$-free multigraphs as the free $2 \mathrm{p}$-algebra [23]. There, graphs have labeled edges, and parallel edges are allowed. We define labeled multigraphs accordingly, with labels in a fixed alphabet $\Sigma$.

Definition 8.1 A (labeled directed) multigraph is a structure $G=\langle V, E, s, t, l\rangle$, where $V$ is a finite type of vertices, $E$ is a finite type of edges, $s, t: E \rightarrow V$ are functions indicating the source and target of each edge, and $l: E \rightarrow \Sigma$ is function indicating the label of each edge. If $G$ is a multigraph, we write $x: G$ to denote that $x$ is a vertex of $G$.

Note that self-loops are allowed, as well as parallel edges with the same label. This definition is rather different from the previous ones for directed and simple graphs: edges are represented explicitly as a (finite) type.

We slightly optimise this definition in Coq by a using a single function with an additional boolean argument to represent the source and target functions:

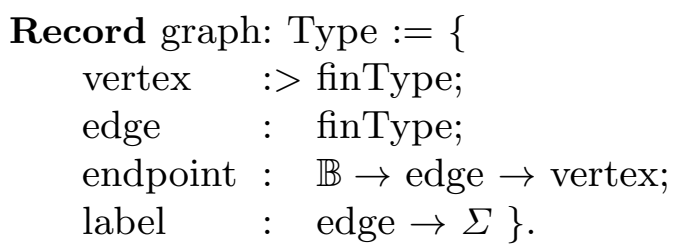

The source function $(s)$ from Definition 8.1 is represented by 'endpoint false'; similarly, the target function is represented by 'endpoint true'. Since source and target functions are often handled in a uniform way, this makes it possible to factor out code and proofs at several places - see, e.g., the code excerpt below defining isomorphisms. For the sake of readability, we stick in this paper to the presentation with two functions, like in Definition 8.1.

Having an explicit set of edges corresponds to the standard representation of graphs in category theory; it makes it possible to use the following notion of isomorphism, where the identity of edges is taken into account.

Definition 8.2 A homomorphism from the graph $G=\langle V, E, s, t, l\rangle$ to the graph $G^{\prime}=\left\langle V^{\prime}, E^{\prime}, s^{\prime}, t^{\prime}, l^{\prime}\right\rangle$ is a pair $\langle f, g\rangle$ of functions $f: V \rightarrow V^{\prime}$ and $g: E \rightarrow E^{\prime}$ that respect the various components: $s^{\prime} \circ g \equiv f \circ s, t^{\prime} \circ g \equiv f \circ t$, and $l \equiv l^{\prime} \circ g$.

An isomorphism is a homomorphism whose two components are bijective functions. We write $G \simeq G^{\prime}$ when there exists an isomorphism between graphs $G$ and $G^{\prime}$.

The corresponding Coq definitions require some care. Indeed, more than the existence of isomorphisms, we often need to keep track of their action on vertices and edges. This is typically the case in the following section, where we work with multigraphs with distinguished vertices. To this end, we define isomorphisms in Type rather than Prop, and we rely on the following notion of bijection between types, where the inverse function is given explicitly. 


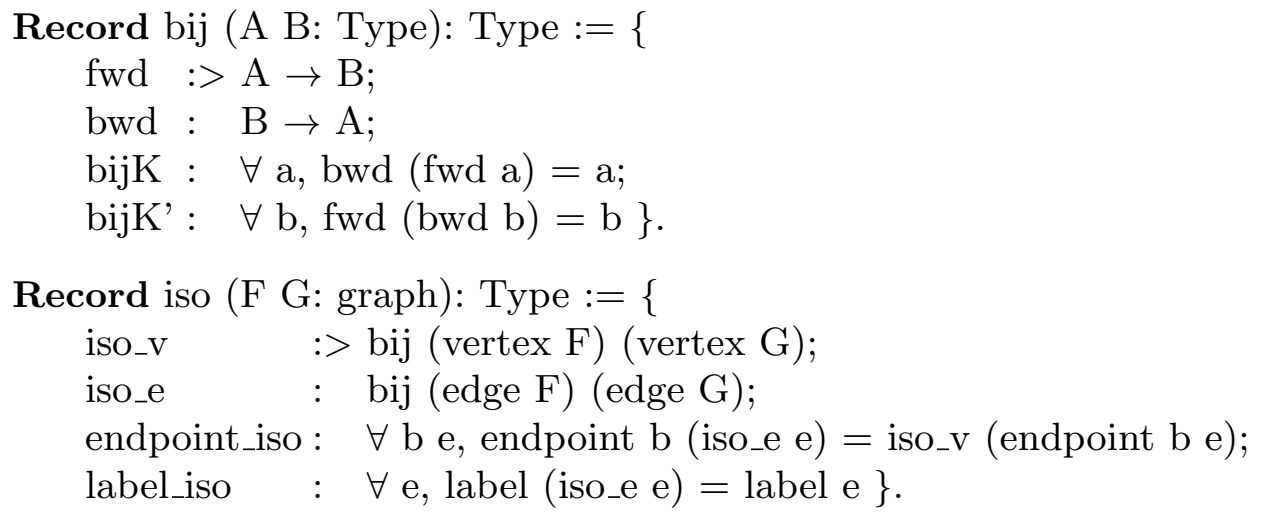

By declaring the first fields of those structures as coercions, we can freely use a bijection as a function, and an isomorphism as a function on vertices. In addition, we set up specific notations to use the inverse of a bijection, as well as the edge component of an isomorphism.

Even though these definitions are computational, it is convenient to think of them as equivalence relations on types and graphs. We use for that the extension of Coq's setoid rewriting tactics [33] to Type valued relations (a proof relevant extension inspired by homotopy type theory [35]).

Remark 8.3 In subsequent work [12], we had to generalize the notions of graphs and isomorphisms presented here, in order to deal with graphs with vertex-labels, and with isomorphisms allowing one to flip edge directions. The Coq development associated to this article, to which the various hyperlinks point to, contains this generalization. As explained in the code, a specific choice of parameters makes it possible to obtain definitions and theorems that are equivalent to the ones presented here.

Every labeled multigraph can be seen as a directed graph by forgetting labels as well as edge identities and multiplicities. By further forgetting edge directions and removing self-loops, we obtain a simple graph which we call the skeleton.

Definition 8.4 Let $G=\langle V, E, s, t, l\rangle$. The skeleton of $\langle V, E, s, t, l\rangle$ is the simple graph $\langle V, R\rangle$ where $x R y$ iff $x \neq y$ and there exists an edge $e: E$ such that $s(e)=x$ and $t(e)=y$ or vice versa.

Skeletons allow us to reuse our definitions and results about simple graphs on multigraphs, e.g., those about minors and treewidth. That taking the skeleton of a graph does not change the type of vertices greatly simplifies lifting properties of the skeleton to the graph and vice versa. In practice, we turn the construction of taking the skeleton into a coercion from multigraphs to simple graphs.

In addition to comparing labeled multigraphs up to isomorphism, we will need to compose them in various ways to show that they form an algebra. This usually involves taking two graphs and merging (or identifying) some vertices in their disjoint union. To this end, we define the generic operations of taking the disjoint union of two graphs and quotienting a graph:

Definition 8.5 Let $G=\langle V, E, s, t, l\rangle$ and $G^{\prime}=\left\langle V^{\prime}, E^{\prime}, s^{\prime}, t^{\prime}, l^{\prime}\right\rangle$. The disjoint union of $G$ and $G^{\prime}$, written $G+G^{\prime}$, is defined to be the graph

$$
\left\langle V+V^{\prime}, E+E^{\prime}, s+s^{\prime}, t+t^{\prime}, l+l^{\prime}\right\rangle
$$


Here, $f+f^{\prime}$ (for $f \in\{s, t, l\}$ ) is the pointwise lifting of $f$ and $f^{\prime}$ to the sum type $E+E^{\prime}$ (with result in $V+V^{\prime}$ or $\Sigma$ ).

Definition 8.6 Let $G=\langle V, E, s, t, l\rangle$ and let $\approx: G \rightarrow G \rightarrow \mathbb{B}$ be an equivalence relation. The quotient of $G$ modulo $\approx$, written $G / \approx$, is defined to be the graph

$$
\left\langle V_{/ \approx}, E, \pi \circ s, \pi \circ t, l\right\rangle
$$

In order to prepare the ground for the following section, we prove several isomorphism lemmas about those operations. Here is a non-exhaustive list:

Lemma 8.7 For all multigraphs $F, F^{\prime}, G, G^{\prime}, H$, we have:

1. $F+G \simeq G+F$ and $F+(G+H) \simeq(F+G)+H$.

2. If $F \simeq G$ and $F^{\prime} \simeq G^{\prime}$, then $F+F^{\prime} \simeq G+G^{\prime}$.

3. If $\approx, \approx^{\prime}$ are two pointwise equivalent equivalence relations on (the vertices) of $F$, then $F_{/} \approx \simeq F_{/} \approx^{\prime}$.

4. If $F \simeq G$ then $F / \approx \simeq G / \approx^{\prime}$, where $\approx^{\prime}$ is the equivalence relation on $G$ induced through the given isomorphism by a given equivalence relation $\approx$ on $F$.

5. $F+G / \approx \simeq(F+G)_{/} \approx^{\prime}$ where $\approx$ is an equivalence relation on $G$ and $\approx^{\prime}$ is its extension to $F+G$ (leaving all vertices of $F$ in singleton classes).

6. $\left(F_{/} \approx\right)_{/ \approx^{\prime}} \simeq F_{/ \approx^{\prime \prime}}$, where $\approx$ is an equivalence relation on $F, \approx^{\prime}$ is an equivalence relation on $F / \approx$, and $\approx^{\prime \prime}$ is the equivalence relation on $F$ obtained by composing $\approx$ and $\approx^{\prime}$.

7. $(F+G)_{/ \approx} \simeq F / \approx$ when $G$ has no edge, $\approx i$ is an equivalence relation on $F+G, \approx^{\prime}$ is its restriction to $F$, and for all $x: G$ there exists $y: F$ with $\operatorname{inr} x \approx \operatorname{inl} y$.

The first three items are straightforward. Item (2) makes it possible to rewrite isomorphims in contexts composed of disjoint unions; Item (4) extends this to rewriting isomorphisms under quotients. The latter illustrates the need to work with computational definitions: the induced equivalence relation $\approx^{\prime}$ depends on the concrete behaviour of the given isomorphism. The last three items are more involved; they make it possible to move quotients out of disjoint unions and to compress them: this is convenient to obtain 'normal forms' for nested quotients (on disjoint unions) occurring in the laws to be proved in the following section.

The proofs for establishing the various points in the above lemma follow the same pattern: we first exhibit the appropriate bijections on the underlying types, and then show that they can be extended into graph isomorphisms.

\section{The 2p-algebra of two-pointed multigraphs}

Using the operations on multigraphs defined in the previous section, we can construct a 2p-algebra [23] whose elements are multigraphs. The syntax of $2 \mathrm{p}$ algebras is as follows; the laws these operations should satisfy are listed below, in Lemma 9.3.

$$
u, v, w::=u \cdot v|u \| v| u^{\circ}|\operatorname{dom}(u)| 1|\top| a \quad(a \in \Sigma)
$$

This syntax makes it possible to denote labeled multigraphs with two designated vertices (the input and the output). For instance, the terms $a \cdot\left(b \| c^{\circ}\right) \| d$ and $1 \| a \cdot b$ will be interpreted below as the following graphs: 


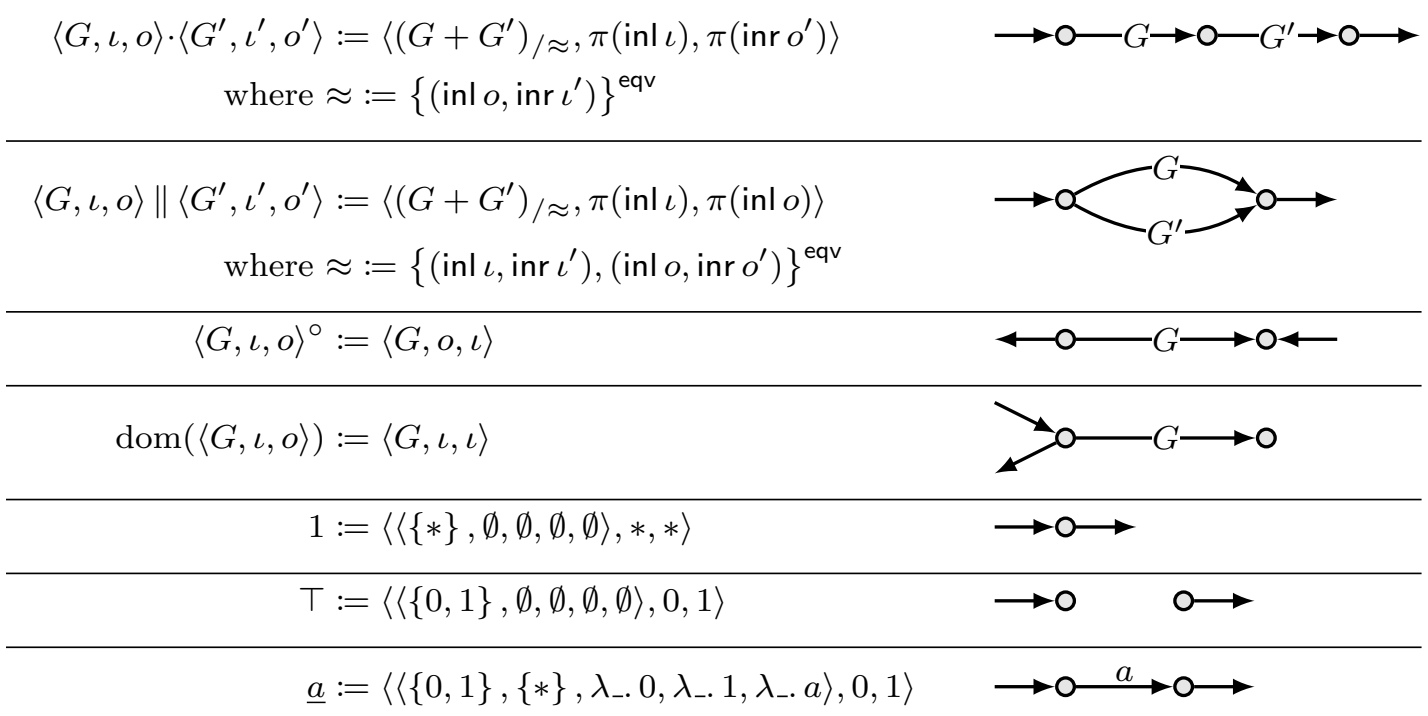

Fig. 7: The algebra of 2p-graphs.
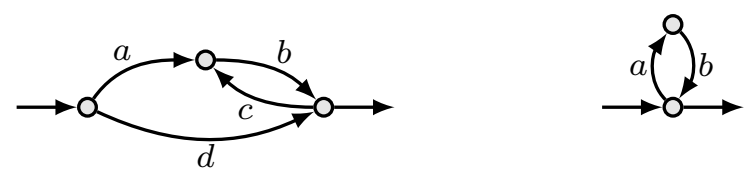

Definition 9.1 A two-pointed graph (or 2p-graph for short) is a structure $\langle G, \iota, o\rangle$ where $G$ is a multigraph and $\iota: G$ and $o: G$ are two vertices called input and output respectively.

The notions of homomorphism and isomorphism are extended accordingly: on $2 \mathrm{p}$ graphs, they should map the input to the input, and likewise for the outputs.

The algebra of 2p-graphs is defined in Figure 7, formally on the left, and informally on the right. The binary operations of the syntax correspond to series and parallel composition. We use the previous operations of disjoint union and quotient in their formal definition, where $A^{\text {eqv }}$ denotes the equivalence relation generated by the pairs in $A$. The first unary operation, converse, exchanges input and output; the second one, domain, relocates the output to the input. The constant 1 represents the graph with just a single vertex; $T$ is the disconnected graph with two vertices (one being the input and the other the output). Letters represent single edges.

As announced above, this algebra allows us to recursively interpret every term $t$ as a 2p-graph $\mathrm{g}(t)$. In particular, the graphs depicted before Definition 9.1 indeed correspond to $\mathrm{g}\left(a \cdot\left(b \| c^{\circ}\right) \| d\right)$ and $\mathrm{g}(1 \| a \cdot b)$. Note that the second graph is also represented by the term $\operatorname{dom}\left(a \| b^{\circ}\right)$.

We remark that the definition of the function $g$ works seamlessly in our setting where graphs are represented using finite types. More precisely, we make use of the fact that the type of vertices is not part of the type of graphs and the fact that finite types are closed under disjoint union and quotients. Defining the function $\mathrm{g}$ in a setting where the vertices of graphs are given as subsets of some fixed type (as is the case in [27] and [32]) would require either a type closed under these operations 
(e.g., hereditarily finite sets as used by Paulson to formalize finite automata in Isabelle/HOL [28]) or an explicit encoding.

Two show that $2 \mathrm{p}$-graphs form a $2 \mathrm{p}$-algebra, we need to prove that the operations preserve isomorphisms, and satisfy the twelve axioms of $2 \mathrm{p}$-algebras [23].

Lemma 9.2 Let $F \simeq F^{\prime}$ and $G \simeq G^{\prime}$. Then we have $F\left\|G \simeq F^{\prime}\right\| G^{\prime}, F \cdot G \simeq F^{\prime} \cdot G^{\prime}$, and $F^{\circ} \simeq\left(F^{\prime}\right)^{\circ}$.

Proof The first two points follow by using items 2 and 4 from Lemma 8.7: parallel and sequential composition are both defined as a quotient of a disjoint union. The third point is straightforward: the given isomorphism can be reused directly.

Lemma 9.3 For all 2p-graphs $F, G, H$, we have

1. $F\|G \simeq G\| F$;

2. $(F \| G)\|H \simeq F\|(G \| H)$;

3. $(F \cdot G) \cdot H \simeq F \cdot(G \cdot H)$;

4. $F \cdot 1 \simeq F$

5. $F^{\circ \circ} \simeq F$;

6. $(F \| G)^{\circ} \simeq F^{\circ} \| G^{\circ}$;

7. $(F \cdot G)^{\circ} \simeq G^{\circ} \cdot F^{\circ}$;

8. $\operatorname{dom}(F) \simeq 1 \| F \cdot T$;

9. $1 \| 1 \simeq 1$;

10. $1 \| F \cdot G \simeq \operatorname{dom}\left(F \| G^{\circ}\right)$;

11. $F \cdot \top \simeq \operatorname{dom}(F) \cdot \top$;

12. $(F \| 1) \cdot G \simeq(F \| 1) \cdot \top \| G$;

(Those laws are equivalent to those of 2p-algebras [23] and slightly easier to prove; that $T$ is a neutral element for parallel composition follows from items 9 and 12.)

Proof All laws are easily checked informally by drawing pictures. Formalizing them however requires some non-trivial work. In particular, laws like associativity of parallel composition (2) do not follow directly from Lemma 8.7(1): parallel and sequential compositions involve quotients in addition to disjoint unions.

For most laws, we use Lemma $8.7(4,5,6)$ in order to rewrite both sides into a single quotient of a disjoint union of the starting graphs. Then we use Lemma 8.7(1,2, $4,7)$ to align the disjoint unions and prune useless components (those appear when the starting expressions contain the constants 1 or $T$ ). We finally conclude by using Lemma 8.7(3) and comparing the obtained equivalence relations.

For item (8) for instance, ignoring the inputs and outputs, the multigraph in the right-hand side is successively rewritten into expresions of the form $(1+(F+2)) / \approx$, $(F+(1+2)) / \approx^{\prime}$, and $F_{/ \approx^{\prime \prime}}$; it then suffices to show that $\approx^{\prime \prime}$ is the discrete equivalence relation. The equivalence relations used to define parallel and sequential compositions are easily described as the equivalence closures of short lists of pairs; we use variants of Lemma 8.7(4-7) maintaining this explicit representation so that it is straightforward to compare the final equivalence relations.

Putting everything together, we obtain:

Theorem 9.4 The set of 2p-graphs form a 2p-algebra (up to isomorphism). 
While the set of all 2p-graphs form a 2p-algebra, we need to restrict to graphs of treewidth at most two to obtain the free $2 \mathrm{p}$-algebra. We show that those form a subalgebra, and in particular that the graph of every term has treewidth at most two. To be more precise, we need to restrict to $2 \mathrm{p}$-graphs whose strong skeleton has treewidth at most two:

Definition 9.5 The (weak) skeleton of a 2 p-graph $\langle G, \iota, o\rangle$ is the skeleton of $G$; its strong skeleton of is the skeleton of $G$ with an additional $\iota$-edge.

The following lemma makes it possible to show that both series and parallel composition preserve treewidth two.

Lemma 9.6 Let $G_{1}=\left\langle G_{1}^{\prime}, \iota, o\right\rangle$ and $G_{2}=\left\langle G_{2}^{\prime}, \iota^{\prime}, o^{\prime}\right\rangle$ be $2 p$-graphs and let $\left\langle T_{i}, B_{i}\right\rangle$ $(i \in\{1,2\})$ be tree decompositions of the strong skeletons of $G_{1}$ and $G_{2}$ respectively. Further let $\approx$ be an equivalence relation on $G_{1}+G_{2}$ identifying at least two vertices from the set $P:=\left\{\operatorname{inl} \iota, \operatorname{inr} \iota^{\prime}, \operatorname{inl} o, \operatorname{inr} o^{\prime}\right\}$ and no other vertices. Then there exists a tree decomposition of the skeleton of $(G 1+G 2) / \approx$ of width at most two having a node $t$ such that $P_{/} \approx \subseteq B(t)$.

Proof We use the three following facts. 1) A tree decomposition for a disjoint union of simple graphs can be obtained by taking the disjoint union of tree decompositions for those graphs. 2) Two trees of a tree decomposition can be joined through a new node containing the vertices of its neighbors. 3) A tree decomposition can be quotiented (to give a tree decomposition of a quotiented graph) as soon as it has nodes for all equivalence classes.

Theorem 9.7 For every term $u$, the strong skeleton of $\mathrm{g}(u)$ has a tree decomposition of width at most two.

Proof By induction on $u$. The cases for $\|$ and f follow with Lemma 9.6. All other cases are trivial.

\section{Checkpoints}

In the following three sections we show that Theorem 9.7 is sharp in the sense that all graphs whose strong skeleton has treewidth two can, up to isomorphism, be constructed as $\mathrm{g}(u)$ for some term $u$. We prove this by defining an extraction function taking as input some $\mathrm{K}_{4}$-free graph and returning some term describing the graph. We focus on connected graphs first: they form a subalgebra when removing $T$ from the signature, and they can be decomposed recursively. We obtain an extraction function handling disconnected components in a second step (Section 12).

Obtaining a recursive function extracting terms from graphs requires a careful analysis of the structure treewidth-two graph. We base our analysis on the excluded minor characterization of the graphs of treewidth at most two. The central tool in this analysis are the singleton separators of the input and the output in (the skeleton of) the input graph. We refer to these as checkpoints since every path from input to output needs to cross each of them. Checkpoints allow us to decompose every graph with distinct input and output into sequential composition of $2 \mathrm{p}$ graphs (Figure 8(a)). If at least two of these components are nontrivial, this allows us to proceed recursively. 
(a)

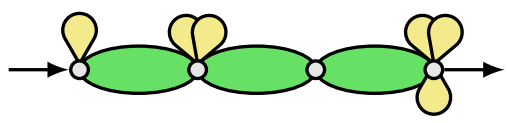

(b)

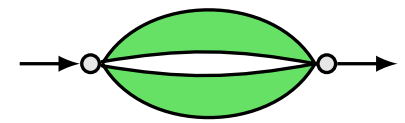

(c)

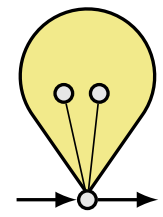

Fig. 8: The three main cases for extracting a term from a $\mathrm{K}_{4}$-free graph.
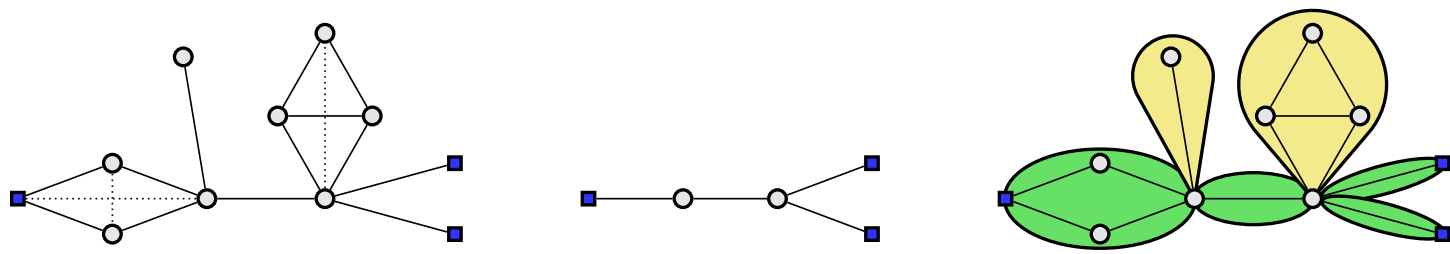

Fig. 9: Link graph, checkpoint graph, and decomposition into intervals and bags.

If there are no proper checkpoints between input and output, we exploit a key property of $\mathrm{K}_{4}$-free graphs: unless the graph consists of just a single edge, in which case the algorithm terminates, it consists of at least two parallel components, making it possible to proceed recursively (cf. Figure 8(b)).

The last case to consider is when the input and the output of the graph coincide. Then the graph either consists only of a single vertex (possibly with self loops) or one can recursively extract a term for the graph obtained by relocating the output to one of the neighbors of the input and use the domain operation to recover the starting graph (cf. Figure 8(c)).

For the remainder of this section, $G$ refers to some connected simple graph.

Definition 10.1 The checkpoints between two vertices $x, y$ are the vertices which every $x y$-path must visit:

$$
\text { ср } x y:=\{z \mid\{z\} \text { is an }\{x\}\{y\} \text {-separator }\}
$$

A checkpoint $z \in \operatorname{cp} x y$ is called proper if $z \notin\{x, y\}$ (i.e., if $\{z\}$ separates $x$ and $y$ ). Two vertices $x, y$ are linked, written $x \diamond y$, when $x \neq y$ and cp $x y=\{x, y\}$, i.e., when there are no proper checkpoints between $x$ and $y$. The link graph of $G$ is the graph of linked vertices.

Note that the link graph of $G$ includes all the edges of $G$. Consider the graph on the left in Figure 9; its link graph is obtained by adding the three dotted edges to the existing ones.

Lemma 10.2 1. ср $x x=\{x\}$

2. $\{x, y\} \subseteq \operatorname{cp} x y=\operatorname{cp} y x$

Definition 10.3 Let $U$ be a set of vertices of $G$. The checkpoints of $U$, written CP $U$, are the vertices which are checkpoints of some pair in $U$.

$$
\mathrm{CP} U:=\bigcup_{x, y \in U} \operatorname{cp} x y
$$


The checkpoint graph of $U$ is the subgraph of the link graph induced by this set. We also denote this graph by CP $U$.

The graph in the middle of Figure 9 is the checkpoint graph of the one of the left, when $U$ consists of the blue square vertices.

Lemma 10.4 Let $x, y \in \mathrm{CP} U$. Then $\operatorname{cp} x y \subseteq \mathrm{CP} U$.

Proof We have $x \in \operatorname{cp} x_{1} x_{2}$ and $y \in \operatorname{cp} y_{1} y_{2}$ for some vertices $\left\{x_{1}, x_{2}, y_{1}, y_{2}\right\} \subseteq U$ by the definition of CP. Fix some $z \in \mathrm{cp} x y$. If $z \in\{x, y\}$, the claim is trivial, so assume $z \notin\{x, y\}$. Hence, we obtain either an $x x_{1}$-path or an $x x_{2}$-path not containing $z$ by splitting some irredundant $x_{1} x_{2}$-path at $x$. Without loss of generality, the $x x_{1}$-path avoids $z$. Similarly, we obtain, again w.l.o.g., a $y y_{1}$-path avoiding $z$. Thus $z \in \operatorname{cp} x_{1} y_{1}$ since the existence of an $x_{1} y_{1}$-path avoiding $z$ would contradict $z \in \operatorname{cp} x y$ (by concatenation with the paths obtained above).

Based on the notion of checkpoint, we define bags and intervals to decompose graphs as depicted on the right of right in Figure 9: the green (oval) components are intervals and the yellow components are bags.

Definition 10.5 Let $x, y: G$. The strict interval $\rrbracket x ; y \llbracket$ is the following set of vertices.

$$
\rrbracket x ; y \llbracket:=\{z \mid y \notin \mathrm{cp} z x \wedge x \notin \mathrm{cp} z y\}
$$

That is $\rrbracket x ; y \llbracket$ contains those vertices that can reach $x$ without passing through $y$ and vice versa. The interval $\llbracket x ; y \rrbracket$ is obtained by adding $x$ and $y$ to that set. We abuse notation and also write $\llbracket x ; y \rrbracket$ for the subgraph of $G$ induced by the set $\llbracket x ; y \rrbracket$.

Definition 10.6 The bag of a checkpoint $x \in \mathrm{CP} U$ is the set of vertices that need to cross $x$ in order to reach the other checkpoints.

$$
\llbracket x \rrbracket_{U}:=\{z \mid \forall y \in \mathrm{CP} U . x \in \mathrm{cp} z y\} .
$$

As before, we also write $\llbracket x \rrbracket_{U}$ for the induced subgraph of $G$.

Note that $\llbracket x \rrbracket_{U}$ depends on $U$ and differs from $\llbracket x ; x \rrbracket$ (which is always the singleton $\{x\}$ ). We first show that distinct bags as well as adjacent bags and strict intervals are disjoint.

Lemma 10.7 1. If $y \in \mathrm{CP} U$, then $\llbracket x \rrbracket_{U} \cap \rrbracket x ; y \llbracket=\emptyset$.

2. If $x, y \in \mathrm{CP} U$ and $x \neq y$, then $\llbracket x \rrbracket_{U} \cap \llbracket y \rrbracket_{U}=\emptyset$.

3. If $z \in \operatorname{cp} x y$, then $\llbracket x ; y \rrbracket=\llbracket x ; z \rrbracket \cup \llbracket z \rrbracket_{\{x, y\}} \cup \llbracket z ; y \rrbracket$.

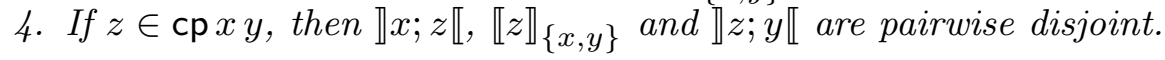

The following proposition is crucial for term extraction; it allows us to split graphs into parallel components provided the graph cannot be split into to sequential components (case (b) in Figure 8; this is the only place in the extraction where $\mathrm{K}_{4}$-freeness of the input graph is being used rather than just being transferred to subgraphs).

Lemma 10.8 Let $\iota, o: G$ such that $G+\iota$ is $\mathrm{K}_{4}$-free and $\iota \diamond o$, but not $\iota-o$. Then $\rrbracket \iota ; o \llbracket$ has at least two connected components. 
Proof Since $\iota \diamond o$, we have that every set $S$ separating $\iota$ and $o$ has size $\geq 2$. By Corollary 4.14, we obtain two independent $\iota$-paths $\pi_{1}$ and $\pi_{2}$ with nonempty interior. Fix $x_{1} \in\left[\pi_{i}\right]$ and $x_{2} \in\left[\pi_{2}\right]$. It suffices to show that $x_{1}$ and $x_{2}$ are not connected in $\rrbracket \iota ; o \llbracket$. Assume there exists an $x_{1} x_{2}$-path $\rho$ in $\rrbracket \iota ; o \llbracket$. Let $z$ be the first vertex on $\rho$ that is in $\left[\pi_{2}\right]$ and let $z^{\prime}$ be the last vertex before $z$. Taking $\pi_{3}$ to be a one-edge $\iota$-path, we obtain the same situation as in Figure 5 (except that $\left[\pi_{3}\right]=\emptyset$, which doesn't influence the proof), so we reuse the construction underlying Lemma 7.1

Remark 10.9 Lemma 10.8 corresponds to [23, Proposition 20(i)], where it is proved by building on a long series of additional lemmas about checkpoints; this is also the approach followed in [10]. Thanks to Corollary 4.14 of Menger's theorem, which we used to prove the minor exclusion theorem for treewidth at most two (Theorem 7.6), these lemmas are no longer required in the present formalization. This should not come as a surprise since thanks to Theorem 9.7, the extraction function we are defining eventually leads to an alternative proof of Theorem 7.6. In a sense, Corollary 4.14 captures the central graph-theoretic argument.

In order to apply Lemma 10.8 to the various intervals in the sequential decomposition of a $2 \mathrm{p}$-graph, we need to show that the strong skeletons induced by these intervals are again $\mathrm{K}_{4}$-free.

Lemma 10.10 Let $\iota, o: G$ such that $G+\iota 0$ is $\mathrm{K}_{4}$-free and let $x, y \in \mathrm{cp} \iota$ such that $x \neq y$. Then $\llbracket x ; y \rrbracket+x y$ is $\mathrm{K}_{4}$-free.

Proof Without loss of generality $x$ appears before $y$ on every $\iota$-path. We obtain that $\llbracket x ; y \rrbracket+x y$ is a minor of $G+\iota o$ by collapsing $\llbracket x \rrbracket_{\{x, y\}}$ (which contains $\iota$ ) to $x$ and $\llbracket y \rrbracket_{\{x, y\}}$ (which contains $o$ ) to $y$. The claim then follows by transitivity of the minor relation.

\section{Extracting Terms from $\mathrm{K}_{4}$-free Graphs}

We say that a 2 p-graph $G$ is $C K 4 F$ if its skeleton is connected and its strong skeleton is $\mathrm{K}_{4}$-free. We now define a function extracting terms from CK4F graphs. Defining this function in Coq is challenging for a number of reasons. First, its definition involves ten cases, most with multiple recursive calls. Second, we need to argue that all the recursive calls are made on smaller graphs which are CK4F.

To facilitate the definition, we construct our own operator for bounded recursion. The reason for this is that none of the facilities for defining functions in Coq (e.g., Fixpoint, Function and Program) are suited to deal with the kind of complex function definition we require. We define a bounded recursion operator with the following type:

$$
\text { Fix }: \forall X Y . Y \rightarrow(X \rightarrow \mathbb{N}) \rightarrow((X \rightarrow Y) \rightarrow X \rightarrow Y) \rightarrow X \rightarrow Y
$$

Here, the argument of type $X \rightarrow \mathbb{N}$ is a measure on the input to bound the number of recursive calls, and the argument of type $Y$ is the default value to be returned when no more recursive calls are allowed.

We only need one lemma about the recursion operator, namely that the operator satisfies the usual fixpoint equation provided that the functional it is applied to 
calls its argument only on smaller arguments in the desired domain of the function (here, CK4F). ${ }^{4}$ That is, we have the following lemma:

$$
\begin{aligned}
\text { Fix_eq } & : \forall(X Y: \text { Type })(P: X \rightarrow \text { Prop })\left(x_{0}: Y\right)(m: X \rightarrow \mathbb{N})(F:(X \rightarrow Y) \rightarrow X \rightarrow Y) . \\
& (\forall f g x . P x \rightarrow(\forall y . P y \rightarrow m y<m x \rightarrow f y=g y) \rightarrow F f x=F g x) \rightarrow \\
& \forall x . P x \rightarrow \text { Fix } x_{0} m F x=F\left(\text { Fix } x_{0} m F\right) x
\end{aligned}
$$

While its proof is straightforward, this lemma is useful in that it allows us to abstract from the fact that we are using bounded recursion (i.e., neither the default result nor the recursion on $\mathbb{N}$ are visible in the proofs).

We now define the extraction function using the recursion operator. The various cases of the definition roughly correspond to the cases outlined in Figure 8. The main difference is that in case (a), rather than partitioning the graph as shown in the picture, we only identify a single nontrivial bag or a single proper checkpoint between input and output. This is sufficient to make recursive calls on smaller graphs. In the case where input and output coincide (case (c)), we relocate the output and proceed recursively. This requires a measure that treats graphs with shared input and output as larger than those with distinct input and output:

Definition 11.1 Let $G=\langle\langle V, E, s, t, l\rangle, \iota, o\rangle$ be a 2p-graph. The measure of $G$ is $2|E|$ if $\iota \neq o$ and $2|E|+1$ if $\iota=o$.

The term extraction function is then defined as

$$
\mathrm{t}:=\text { Fix } 1 \text { measure } \mathrm{F}
$$

where the definition of $F$ is given in Figure 10. This definition makes use of a number of auxiliary constructions which we define below. For a set of vertices $U$ and a set of edges $E$ (of some graph $G$ ) such that $\{s(e), t(e)\} \subseteq U$ for all $e$, the subgraph of $G$ with vertices $U$ and edges $E$ is written $G[U, E]$. We write $\mathcal{E}(U)$ for the set of edges with source and target in $U$ and the induced subgraph for $U$, written $G[U]$, is defined as $G[U, \mathcal{E}(U)]$. For 2p-graphs $G, G[U]$ and $G[U, E]$ are only defined if $\{\iota, o\} \subseteq U$. In this case, $G[U]$ and $G[U, E]$ have the same input and output as $G$.

When instantiating the definitions above, $U$ will sometimes be an interval or a bag. In this case, the intervals and bags are computed on the weak skeleton of $G$ (not the strong skeleton). For a given 2p-graph $G=\left\langle G^{\prime}, \iota, o\right\rangle$, we also define:

$$
\begin{aligned}
\text { components }(U) & :=\{C \mid C \text { connected component of } U \text { in the skeleton of } G\} \\
\text { component }(C) & :=G[C \cup\{\iota, o\}] \\
\text { redirect }(C) & :=\left\langle G^{\prime}[C \cup\{\iota\}], \iota, x\right\rangle \text { where } x \text { is some neighbor of } \iota \text { in } C \\
G[x, y] & :=\left\langle G^{\prime}[\llbracket x ; y \rrbracket, \mathcal{E}(\llbracket x ; y \rrbracket) \backslash(\mathcal{E}(\{x\}) \cup \mathcal{E}(\{y\})]), x, y\right\rangle \\
G[x] & :=\left\langle G^{\prime}\left[\llbracket x \rrbracket_{\{\iota, o\}}\right], x, x\right\rangle \\
\operatorname{tm}(e) & := \begin{cases}l(e) & s(e)=\iota \wedge t(e)=o \\
l(e)^{\circ} & \text { otherwise }\end{cases}
\end{aligned}
$$

Note that component $(C)$ is obtained as an induced subgraph of $G$ whereas the other constructions are obtained as subgraphs of $G^{\prime}$ (with new inputs and outputs).

Before we can establish properties of $t$, we need to establish that all (relevant) calls to $t$ in $\mathrm{F}$ are made on $\mathrm{CK} 4 \mathrm{~F}$ graphs with smaller measure.

\footnotetext{
4 To be precise, the functional may call its argument on anything. However, the result may
} only depend on calls to smaller arguments in the domain. 


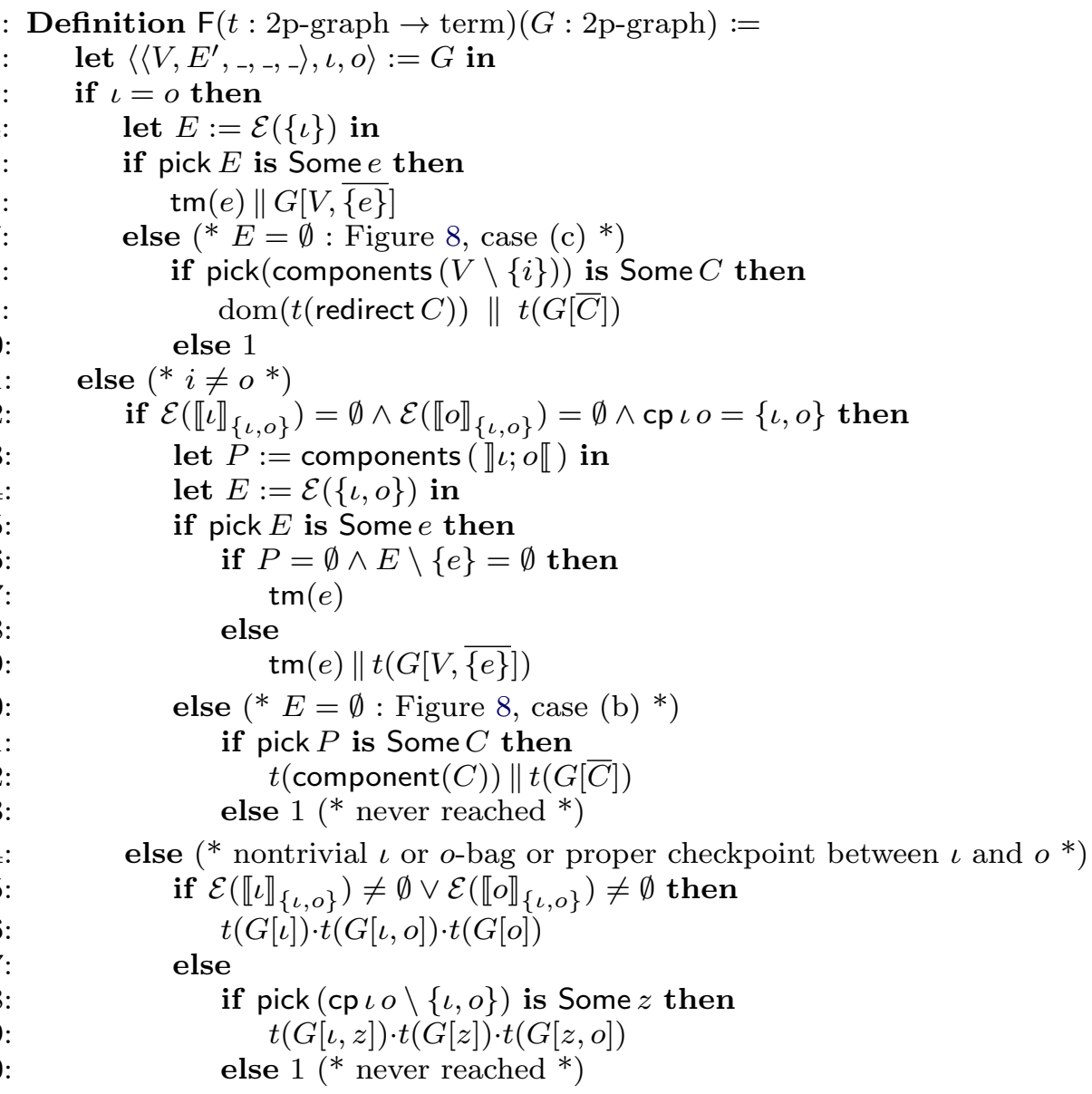

Fig. 10: The term extraction function

Lemma 11.2 Let $t, t^{\prime}$ be functions from graphs to terms. If $t$ and $t^{\prime}$ agree on all $C K 4 F$ graphs with measure smaller than a $C K 4 F$ graph $G$, then $\mathrm{F} t G=\mathrm{F} t^{\prime} G$.

The proof of this lemma boils down to a number of lemmas for the various branches of F. For each recursive call, we need to establish both that the measure decreases and that the graph is indeed CK4F. When splitting of a parallel component (line 22), Lemma 10.8 ensures that there are at least two nonempty components, thus ensuring that the remainder of the graph is both smaller and connected. Note that the case distinction in line 16 is required since if $P=\emptyset$, removing the last ıo-edge disconnects the graph (the remaining graph would be isomorphic to $T$ ). In the case where there is a proper checkpoint $z$ between input and output (line 29), Lemma 10.10 ensures that the strong skeletons of $G[\iota, z]$ and $G[z, o]$ are $\mathrm{K}_{4}$-free.

As a consequence of Lemma 11.2, we obtain:

Lemma 11.3 Let $G$ be $C K 4 F$. Then $\mathrm{t} G=\mathrm{Ft} G$.

We finally show that interpreting the term extracted from a 2 p-graph $G$ yields a graph that is isomorphic to $G$. As was the case with the formal proof of Theorem 9.4, the difference between what one would find in a detailed paper proof and what is required in order to obtain a formal proof is considerable: the various isomorphisms have to be constructed explicitly in the formal proof while they are left to the reader in a paper proof. 
The extraction function decomposes the graph into smaller graphs in order to extract a term. The interpretation of this term then joins the graphs extracted by the recursive calls back together using the graph operations $\|$ and $\cdot$. We need to establish that the decomposition performed during extraction is indeed correct (i.e., that no vertices or edges are lost or misplaced). This requires establishing a number of additional isomorphism properties.

Correctness of the extraction function is established by induction on the termination measure of the extraction function and follows the case distinctions made during extraction. Most cases boil down to showing that (under the conditions of the respective case) the graph can be expressed in terms of $2 \mathrm{p}$-operations applied to various (pointed) subgraphs. For instance, for line 26 we show that $G \simeq G[\iota] \cdot G[\iota, o] \cdot G[o]$ whenever $\iota \neq o$.

Note that the quotient in $G[\iota] \cdot G[\iota, o]$ merely identifies the vertex $\iota$ in $G[\iota]$ with $\iota$ in $G[\iota, o]$ and that this is the only overlap between the two subgraphs. Consequently, the quotient of the two subgraphs can be replaced by the subgraph obtained using the union of the respective vertex and edge sets. Applying the same reasoning to the other sequential operation leaves us with the subgraph of the full vertex and edge set, which is trivially isomorphic to the whole graph. A similar argument applies to parallel composition, allowing us to establish, among others, the following lemmas with relative ease:

Lemma 11.4 Let $G=\left\langle G^{\prime}, \iota, o\right\rangle$ such that $\iota \neq o$ and the skeleton of $G$ is connected. Then $G \simeq G[\iota] \cdot G[\iota, o] \cdot G[o]$.

Lemma 11.5 Let $G=\left\langle G^{\prime}, \iota, o\right\rangle$ such that $\mathcal{E}\left(\llbracket \iota \rrbracket_{\{\iota, o\}}\right)=\emptyset, \mathcal{E}\left(\llbracket o \rrbracket_{\{\iota, o\}}\right)=\emptyset$, and $\iota \neq o$, and let $z \in \mathrm{cp} \iota o \backslash\{\iota, o\}$. Then $G \simeq G[\iota, z] \cdot G[z] \cdot G[z, o]$.

Lemma 11.6 Let $G=\left\langle G^{\prime}, \iota, o\right\rangle$ with $\mathcal{E}(\{\iota, o\})=\emptyset$ and let $C \in \operatorname{components}(\overline{\{\iota, o\}})$. Then $G \simeq$ component $(C) \| G[\bar{C}]$.

Lemma 11.7 Let $G=\left\langle G^{\prime}, \iota, o\right\rangle$ with $\iota \neq o$ with vertex type $V$ and let $e \in \mathcal{E}(\{\iota, o\})$. Then $G \simeq \mathrm{g}(\operatorname{tm}(e)) \| G[V, \overline{\{e\}}]$.

Theorem 11.8 Let $G$ be $C K 4 F$. Then $\mathrm{g}(\mathrm{t} G) \simeq G$.

Proof By induction on the measure of $G$. We use Lemma 11.3 to unfold the definition of $t$. Each of the cases follows with the induction hypothesis (using the lemmas underlying the proof of Lemma 11.2 to justify that that the induction hypothesis applies) and some isomorphism lemmas (e.g., the lemmas above)

Note that Lemma 11.6 justifies both the split in line 9 and the split in line 22 (in the latter case $\rrbracket \iota ; o \llbracket=\overline{\{\iota, o\}}$ ). We remark that the elimination of quotients on overlapping subgraphs outlined above makes use of the compositional infrastructure for graph isomorphisms developed in Section 8, which was not part of [10]. As a consequence, the correctness argument presented here is significantly simpler than the one in [10] (400 lines instead of 730).

\section{Handling disconnected graphs}

Once we have obtained terms for graphs that are CK4F, it is straightforward to extend this result to (possibly) disconnected graphs. We define a function $\mathrm{t}^{\top}$ extracting terms from arbitrary $2 \mathrm{p}$-graphs with $\mathrm{K}_{4}$-free strong skeleton. 
Definition 12.1 If $x: G$, we write $G_{x}$ for the subgraph induced by the connected component of $x$ (in $\mathrm{G}$ ) with input and output set to $x$. We slightly abuse notation and also write $G_{x}$ for the underlying set of vertices of $G$. We then define:

$$
\mathrm{t}^{\top}(G):= \begin{cases}\top \cdot \mathrm{t}\left(G_{x}\right) \cdot \top \| \mathrm{t}^{\top}\left(G\left[\overline{G_{x}}\right]\right) & x \notin G_{\iota} \cup G_{o} \\ \mathrm{t}\left(G_{\iota}\right) \cdot \top \cdot \mathrm{t}\left(G_{o}\right) & \iota \text { and } o \text { disconnected } \\ \mathrm{t}(G) & \text { otherwise }\end{cases}
$$

The three cases are to be read as ordered. That is, if there exists some $x \notin G_{\iota} \cup G_{O}$ we arbitrarily choose one; if not, we check whether $\iota$ and $o$ disconnected and make the appropriate calls to $\mathrm{t}$. The function $\mathrm{t}^{\top}$ terminates since $G\left[\overline{G_{x}}\right]$ has fewer vertices than $G$.

Theorem 12.2 Let $G$ be a 2p-graph with $\mathrm{K}_{4}$-free strong skeleton. Then $\mathrm{g}\left(\mathrm{t}^{\top}(G)\right) \simeq G$.

Proof By induction on $|G|$. If $G$ has some component $C$ containing neither $\iota$ nor $o$, it suffices to show $\top \cdot G[C] \cdot \top \| C[\bar{C}] \simeq G$, which follows with Lemma 9.3. Similarly, we have $G_{\iota} \cdot \top \cdot G_{O} \simeq G$ whenever $G_{\iota}$ and $G_{O}$ are distinct and there are no other components.

Recall that $\mathrm{g}(u)$ has treewidth at most two for all terms $u$ (Theorem 9.7). Hence, Theorem 12.2 provides an alternative proof of Theorem 7.6. Note however, that the two theorems share essential constructions. As noted in Section 10, Menger's Theorem and the construction of $\mathrm{K}_{4}$ from three independent paths (Figure 5) are shared between the two results (cf. Remark 10.9).

\section{Directions for future work}

We presented a library for graph theory built using Coq and the mathematical components library. The library contains formalizations of several interdependent results: Menger's Theorem [24,18], the excluded-minor characterization for treewidth two [13], and a characterization of $\mathrm{K}_{4}$-free $2 \mathrm{p}$-graphs through the syntax of 2 p-algebra [23,11]. For the latter characterization, we managed to use Menger's Theorem to simplify the analysis of checkpoint graphs.

Concerning the completeness of the axioms of $2 \mathrm{p}$-algebras for isomorphism of $2 \mathrm{p}$-graphs $(\dagger)$, we managed during the reviewing process of this article to formalize the proof from [11] - in the connected case. This proof relies on a terminating and confluent rewrite system on term-labeled 2p-graphs. To simplify the formalization, confluence is proved using an open representation of graphs, i.e., where the vertices of a graph are given as finite sets of a fixed countably infinite type, and then transferred to the typed graphs used in the rest of the development. We describe this work in [12]. A natural follow-up would be to also use the rewriting system to extract terms from graphs: this approach is simpler on paper than the one we presented here, but it poses some difficulties when it comes to formalization.

The library keeps evolving, and many results from graph theory would be interesting to formalize as a next step. The excluded-minor characterizations of planar graphs [21] and outer-planar graphs [8] are natural candidates. Further, it would be nice to combine our library with other recent formalizations of graph-theory results. Severín [31] formalized the Cockayne-Hedetniemi Domination Chain using 
a definition of simple graphs closely matching ours, proposing to add this result to our library. Singh and Natarajan [32] formalized the weak perfect graph theorem. They use an open representation for simple graphs where the type of vertices is a parameter of the graph type and the vertices of a graph are given as a subset of this vertex type. Hence, including their result would require to either adapt their proofs to our setting or establish the correspondence between the two representations. This would be similar to the transfer of confluence from one representation of multigraphs to the other we carry out in [12].

A different direction would be to use the library for the verification of graph algorithms. For instance, we do not currently provide functions for computing the treewidth of a graph or to check for graph isomorphisms. In the same vein, it would be nice to develop certified implementations of standard graph algorithms. It should be noted that the definitions in the library are geared towards declarative proofs and not towards the extraction of efficient code. We envision verifying algorithms in this abstract setting and then applying algorithmic and data refinements along the lines of [5].

Acknowledgements. We would like to thank Guillaume Combette, with whom we developed the first version of the library. We are also grateful to Nicolas Trotignon for his wonderful insights on graph theory.

This is a pre-copyedit version of an article published in the Journal of Automated Reasoning. The final authenticated version is available online at: http://dx.doi . org/10.1007/s10817-020-09543-2

\section{References}

1. C. Chekuri and A. Rajaraman. Conjunctive query containment revisited. Theoretical Computer Science, 239(2):211-229, 2000.

2. C. Chou. A formal theory of undirected graphs in higher-order logic. In Proc. TPHOL, volume 859 of Lecture Notes in Computer Science, pages 144-157. Springer, 1994.

3. C.-T. Chou. Mechanical verification of distributed algorithms in higher-order logic*. The Computer Journal, 38(2):152-161, 011995.

4. C. Cohen. Pragmatic quotient types in Coq. In Proc. ITP, volume 7998 of Lecture Notes in Computer Science, pages 213-228. Springer, 2013.

5. C. Cohen, M. Dénès, and A. Mörtberg. Refinements for free! In G. Gonthier and M. Norrish, editors, Certified Programs and Proofs (CPP 2013), volume 8307 of Lecture Notes in Computer Science, pages 147-162. Springer, 2013.

6. B. Courcelle. The monadic second-order logic of graphs. I: Recognizable sets of finite graphs. Information and Computation, 85(1):12-75, 1990.

7. B. Courcelle and J. Engelfriet. Graph Structure and Monadic Second-Order Logic - A Language-Theoretic Approach, volume 138 of Encyclopedia of mathematics and its applications. Cambridge University Press, 2012.

8. R. Diestel. Graph Theory (2nd edition). Graduate Texts in Mathematics. Springer, 2000.

9. C. Doczkal, G. Combette, and D. Pous. Coq formalization accompanying this paper. https://perso.ens-lyon.fr/damien.pous/covece/graphs/.

10. C. Doczkal, G. Combette, and D. Pous. A formal proof of the minor-exclusion property for treewidth-two graphs. In J. Avigad and A. Mahboubi, editors, Interactive Theorem Proving (ITP 2018), volume 10895 of Lecture Notes in Computer Science, pages 178-195. Springer, 2018.

11. C. Doczkal and D. Pous. Treewidth-two graphs as a free algebra. In Proc. MFCS, volume 117 of LIPIcs, pages 60:1-60:15. Schloss Dagstuhl - Leibniz-Zentrum für Informatik, 2018. 
12. C. Doczkal and D. Pous. Completeness of an axiomatization of graph isomorphism via graph rewriting in Coq. In Proc. Proc. of 9th ACM SIGPLAN International Conference on Certified Programs and Proofs (CPP '20), January 20-21, 2020, New Orleans, LA, USA, 2020.

13. R. Duffin. Topology of series-parallel networks. Journal of Mathematical Analysis and Applications, 10(2):303-318, 1965.

14. J. Dufourd and Y. Bertot. Formal study of plane Delaunay triangulation. In Proc. ITP, volume 6172 of Lecture Notes in Computer Science, pages 211-226. Springer, 2010.

15. E. C. Freuder. Complexity of k-tree structured constraint satisfaction problems. In Proc. NCAI, pages 4-9. AAAI Press / The MIT Press, 1990.

16. P. Freyd and A. Scedrov. Categories, Allegories. North Holland. Elsevier, 1990.

17. G. Gonthier. Formal proof - the four-color theorem. Notices Amer. Math. Soc., 55(11):1382-1393, 2008.

18. F. Göring. Short proof of menger's theorem. Discrete Mathematics, 219(1-3):295-296, 2000.

19. M. Grohe. The complexity of homomorphism and constraint satisfaction problems seen from the other side. Journal of the ACM, 54(1):1:1-1:24, 2007.

20. P. Hall. On representatives of subsets. J. London Math. Soc., 10:26-30, 1935.

21. K. Kuratowski. Sur le problème des courbes gauches en topologie. Fund. Math., 15:271283, 1930. in French.

22. P. Lammich and S. R. Sefidgar. Formalizing the Edmonds-Karp algorithm. In J. C. Blanchette and S. Merz, editors, Interactive Theorem Proving (ITP 2016), volume 9807 of Lecture Notes in Computer Science, pages 219-234. Springer, 2016.

23. E. C. Llópez and D. Pous. K4-free graphs as a free algebra. In Proc. MFCS, volume 83 of LIPIcs, pages 76:1-76:14. Schloss Dagstuhl - Leibniz-Zentrum für Informatik, 2017.

24. K. Menger. Zur allgemeinen kurventheorie. Fund. Math., pages 96-115, 1927.

25. Y. Nakamura and P. Rudnicki. Euler circuits and paths. Formalized Mathematics, 6(3):417-425, 1997.

26. T. Nipkow, G. Bauer, and P. Schultz. Flyspeck I: tame graphs. In Proc. IJCAR, volume 4130 of Lecture Notes in Computer Science, pages 21-35. Springer, 2006.

27. L. Noschinski. A graph library for Isabelle. Mathematics in Computer Science, 9(1):23-39, 2015.

28. L. C. Paulson. A formalisation of finite automata using hereditarily finite sets. In A. P. Felty and A. Middeldorp, editors, Automated Deduction (CADE-25), volume 9195 of Lecture Notes in Computer Science, pages 231-245. Springer, 2015.

29. D. Pous and V. Vignudelli. Allegories: decidability and graph homomorphisms. In Proc. LiCS, pages 829-838. ACM, 2018.

30. N. Robertson and P. Seymour. Graph minors. XX. Wagner's conjecture. Journal of Combinatorial Theory, Series B, 92(2):325 - 357, 2004.

31. D. E. Severín. Formalization of the domination chain with weighted parameters (short paper). In J. Harrison, J. O'Leary, and A. Tolmach, editors, Interactive Theorem Provin (ITP 2019), volume 141 of LIPIcs, pages 36:1-36:7. Schloss Dagstuhl - Leibniz-Zentrum für Informatik, 2019.

32. A. K. Singh and R. Natarajan. A constructive formalization of the weak perfect graph theorem. In Proc. Proc. of 9th ACM SIGPLAN International Conference on Certified Programs and Proofs (CPP '20), January 20-21, 2020, New Orleans, LA, USA, 2020.

33. M. Sozeau. A new look at generalized rewriting in type theory. J. Form. Reason., 2(1):41$62,2009$.

34. The Mathematical Components team. Mathematical components, 2017.

35. T. Univalent Foundations Program. Homotopy Type Theory: Univalent Foundations of Mathematics. http://homotopytypetheory.org/book, Institute for Advanced Study, 2013. 